\title{
DUALITY: A Simple Formalism for the Analysis of UNITY
}

\section{Rutger M. Dijkstra}

Dept. of Computing Science, University of Groningen, Groningen, Netherlands

Keywords: UNITY logic; Predicate transformer semantics; Demonic and angelic nondeterminism

\begin{abstract}
The 'programming' language DUALITY is presented and its utility for the description and analysis of UNITY is demonstrated. DUALITY is a simple language supporting demonic and angelic choice and two forms of tailrecursion. Starting from the predicate transformer semantics that was developed by C.S. Jutla, E. Knapp, and J.R. Rao for UNITY, we show that, given a UNITY program, we can write a program in DUALITY that has the same formal characteristics. This we use to reformulate and reprove some important theorems from the UNITY theory, viz. the 'PSP-rule' and the 'completion rule'. Finally we show that the predicate transformers proposed by K.M. Chandy and B.A. Sanders for reasoning about concurrent computation also fit nicely within this framework.
\end{abstract}

\section{Introduction}

The contents of this paper are the results of an investigation that started with an attempt to prove a single theorem from UNITY. Although the ambition was modest in the beginning, the project soon grew in scope, yielding, in the end, a kind of cross from various works in the field of predicate transformer semantics. In order to enable the reader to put the contents of this paper in the proper context, we present a short overview of these works.

- In [DiS90] E.W. Dijkstra and C.S. Scholten present what can be considered 'the mother of all predicate transformer semantics'. Abstracting from input 
and output, a computation is regarded as a change of the state of a machine. The authors define the semantics of a statement $S$ to be a pair of predicate transformers, wp.S (weakest precondition) and wlp.S (weakest liberal precondition), which have the operational interpretation that, for any predicate $q$ on the states:

wp.S.q : holds in those initial states for which each execution of $S$ has a final state satisfying $q$.

wlp.S.q : holds in those initial states for which each finite execution of $S$ has a final state satisfying $q$.

Since these predicate transformers give no information about anything but the machine state upon termination, we call this kind of program semantics 'postcondition semantics'.

The book gives the postcondition semantics of a language in which programs are built from primitives using sequential composition, alternative constructs, and iteration. This language is often called 'Dijkstra's guarded command language'.

- In his thesis [Luk90], J.J. Lukkien introduces predicate transformers with an expressiveness that is somewhat higher than the postcondition semantics. Particularly, he introduces, among others, the predicates wev.S.p.q and wlev.S.p.q that have the following operational interpretation:

wev.S.p.q : holds in those initial states for which each execution of $S$ has some intermediate state satisfying $p$ or a final state satisfying $q$.

wlev.S.p.q : holds in those initial states for which each finite execution of $S$ has some intermediate state satisfying $p$ or a final state satisfying $q$.

In contrast to the postcondition semantics, these predicate transformers do contain information about what may happen in the course of execution. For this reason we call this approach 'runtime semantics'.

In his thesis, Lukkien gives the runtime semantics for Dijkstra's guarded command language.

Note that, since no intermediate state satisfies false, the interpretations dictate that wev.S.false $=$ wp. $S$ and wlev.S.false $=$ wlp.S. Thus, the runtime semantics subsumes the postcondition semantics.

- In order to cope with the semantics of recursion, W.H. Hesselink has introduced a programming formalism that is somewhat simpler than Dijkstra's guarded command language[Hes92]. The construction principles of what we call 'Hesselink's recursion language' are: sequential composition, nondeterministic choice, and (possibly mutual) recursion. Assuming a rich enough repertoire of primitives, Hesselink's recursion language subsumes Dijkstra's guarded command language.

In his book Hesselink gives the postcondition semantics for his recursion language and in subsequent articles he generalizes this in two different directions.

- The machinery developed in [Hes92] to deal with the postcondition semantics of recursion can also be used to generate the runtime semantics of Hesselink's recursion language. This is done in [HeR92] in collaboration with R. Reinds.

- R.J.R. Back and J. von Wright [BvW90] introduce the 'demonic choice' and the 'angelic choice'. The former equals Hesselink's nondeterministic choice and the latter is its lattice-theoretical dual. In [Hes94] Hesselink gives the 
postcondition semantics for the extension of his recursion language with the angelic choice.

Since the angelic choice does not have an operational interpretation in the context of programs, the interpretation of the predicates wp.S.q and wlp.S.q is no longer adequate. Following a suggestion from Back and von Wright, Hesselink 'reinterprets' these predicates in the context of a game played by an angel and a demon - hence the vocabulary.

However, it should be stressed that it is programs that we are interested in and not games. Even if the angelic choice defies operational interpretation, its importance lies in the utility in the formal analysis of programming problems.

Insofar as it has bearing on the material to be presented in this paper, the above five points summarize the state of the art in the field of predicate transformer semantics for sequential programs. Now we turn our attention to the relevant formalism(s) for dealing with concurrency.

- In [ChM90] K.M. Chandy and J. Misra present the programming 'package' UNITY that is designed for the construction of parallel programs. UNITY is a package that consists of an extremely simple programming language and a logic for proving properties of the programs. Although the language is simple, it is possible to translate about every program (parallel or not) into it. After this translation, the program can be seen as a - highly nondeterministic infinite repetition and the UNITY logic can be used for proving properties this repetition may enjoy.

The two most important ingredients of the logic are the relations unless and $\mapsto$ ('leads_to') on predicates that have the following interpretation:

$q$ unless $p \quad$ : Once $q$ holds, it will remain true as long as $p$ does not hold. $q \mapsto p \quad:$ Whenever $q$ holds, eventually $p$ will hold.

The relation unless captures safety and the relation $\mapsto$ captures progress.

- Like Hoare-logic, the logic of Misra and Chandy is centred around relations on predicates. In their article [JKR89], C.S. Jutla, E. Knapp and J.R. Rao present a formalism for reasoning about UNITY programs that is equivalent to that of Misra and Chandy, but is entirely in terms of predicate transformers. The two most important of these are the predicate transformers 'wsafe' and 'wlt' that can be interpreted as:

wsafe.q.p : holds in exactly those (initial) states for which it is guaranteed that $q$ holds at least as long as $p$ does not, i.e. 'exit from $q$ ' is possible only 'via $p$ '.

wlt. $p \quad$ : holds in exactly those (initial) states for which it is guaranteed that $p$ will eventually hold.

We put the adjective 'initial' between brackets because each intermediate state is the initial state of the remaining computation.

The predicate transformers wsafe and wlt can be used to replace the relations unless and $\mapsto$ of Misra and Chandy.

The 'programming' language DUALITY to be presented in this paper is designed for a single purpose: the analysis of UNITY - and hence parallelism in the wider context of predicate transformer semantics.

DUALITY has a (kind of) runtime semantics and the language has both demonic and angelic choice. Thus, the language stands somewhere between Hes- 
selink's recursion language with runtime semantics and the extended version with postcondition semantics. However, DUALITY has only two forms of recursion that are barely more general than iteration.

We probably could have accommodated general recursion. The reason for not doing so is entirely pragmatic: we don't need it. Every ingredient of the language DUALITY is present for (at least) one of two reasons: necessity in the analysis of UNITY, or symmetry.

We end this section with an overview of the rest of this paper. For each of the remaining sections, we give a short description of its contents.

1. A preamble, presenting some facts about fixpoints in the general setting of lattice theory.

2. The definition of DUALITY.

3. A very short section highlighting the symmetry of the language.

4. After a brief introduction into UNITY in terms of the predicate transformers from Jutla, Knapp, and Rao, we give the translation of a UNITY program into DUALITY.

5. DUALITY from a lattice theoretical point of view.

6. A very short section on a simple property that we encounter several times.

7. Closes a gap in the argumentation of Section 4.

8. Introducing a safety notion in DUALITY.

9. The same notion for UNITY.

10. Presenting the proofs of some wellknown theorems from UNITY logic.

11. Applying the results to the predicate transformers that Chandy and Sanders propose for reasoning about UNITY programs.

12. Conclusion.

A. Appendix: about an operational model for DUALITY.

B. Appendix: containing a proof that we considered too long to include in the paper itself.

\section{Preamble}

In this section we present some facts from lattice theory. Throughout this section $(L, \leq)$ is an arbitrary complete lattice and $M$ is the set of monotonic functions from and to $L$. As usual, we lift the order on $L$ to $M$ by pointwise extension :

(0) $f \leq \mathrm{g} \equiv(\forall p: p \in L: f . p \leq g \cdot p)$ for all $f, g \in M$.

With this order, $M$ is also a complete lattice.

We use, in all lattices, $L$ and $\sqcap$ to denote suprema and infima respectively, and we use these operators both in infix notation and in quantifier format. The functional application operator, ', is given the highest binding power of all operators and the functional composition operator, ' $o$ ', is given the next highest binding power. We use 'id' for the identity function.

The facts in this section are presented without proof. 


\subsection{On Fixpoints}

For any $f \in M$ we use $\mu f$ to denote $f$ 's least fixpoint and $\boldsymbol{v} f$ to denote $f$ 's greatest fixpoint. In order not to be forced to use explicit functional abstraction we also use the more extended notations $(\boldsymbol{\mu} x:: f . x)$ and $(\boldsymbol{v} x:: f . x)$.

The first result we list is the theorem of Knaster-Tarski:

For any $p \in L$ and $f \in M$ we have :

$f . p=p \Leftarrow \mu f=p \quad$ '(un-)folding',

$f . p \leq p \Rightarrow \mu f \leq p \quad$ 'extremity'.

and similarly

$p=f . p \Leftarrow p=v f \quad$ '(un-)folding',

$p \leq f . p \Rightarrow p \leq v f \quad$ 'extremity'.

Viewing $\mu$ and $\boldsymbol{v}$ as operators from $M$ to $L$ we have

$\mu$ and $v$ are monotonic.

Note that from (2) it follows that, for $f: L^{2} \rightarrow L$ monotonic in both arguments, the expressions $(\mu x:: f .(x, y))$ and $(v x:: f .(x, y))$ are monotonic in $y$.

Now, let ' - ' be a unary operator on $L$ such that for all $p$ and $q$

$$
p \leq q \Rightarrow-q \leq-p \text { and }--p=p,
$$

i.e. ' - ' is an anti-monotonic involution. Then the operator ' - ' is an isomorphism between the lattice and its dual and we have

$$
\begin{aligned}
& \frac{\text { fixpoint inversion rule }}{(\mu x::-f .(-x))=-(v y:: f . y) \text { and }} \\
& (v y::-f .(-y))=-(\mu x:: f . x) \quad \text { for all } f \in M \text {. }
\end{aligned}
$$

\subsection{On Closure and Interior Functions}

For any funtion $f$ from and to $L$ we define:

$f$ is idempotent

$$
\begin{aligned}
& \equiv f \circ f=f . \\
& \equiv p \leq f . p \text { for all } p \in L . \\
& \equiv f . p \leq p \text { for all } p \in L .
\end{aligned}
$$

$f$ is expanding

$f$ is contracting

$f$ is a closure function $\equiv f$ is monotonic, idempotent, and expanding.

$f$ is an interior function $\equiv f$ is monotonic, idempotent, and contracting. Monotonicity being given for the elements of $M$, the properties 'being a closure function' and 'being an interior function' can be expressed more succinctly:

(4) $f$ is a closure function $\equiv f \circ f \sqcup i d \leq f$ and

$f$ is an interior function $\equiv f \leq f \circ f \sqcap i d$ for all $f \in M$.

For any $f$ in $M$ we define 'the closure of $f$ ' cl. $f$ (also in $M$ ). The reader may take any of the following four characterizations as definition and consider the other three to be theorems.

cl. $f$ is the least closure function that is at least $f$, cl. $f=(\boldsymbol{\mu} g:: g \circ g \sqcup i d \sqcup f)$, 


$$
\begin{aligned}
& \text { cl. } f=(\boldsymbol{\mu} g:: f \circ g \sqcup i d), \\
& \text { cl. } f . p=(\boldsymbol{\mu} x:: f . x \sqcup p) .
\end{aligned}
$$

Similarly we define 'the interior of $f$ ' int. $f$ for any $f$ in $M$, such that:

$$
\begin{aligned}
& \text { int. } f \text { is the greatest interior function that is at most } f \text {, } \\
& \text { int. } f=(v g:: g \circ g \sqcap i d \sqcap f), \\
& \text { int. } f=(v g:: f \circ g \sqcap i d), \\
& \text { int.f. } p=(v x:: f . x \sqcap p) \text {. }
\end{aligned}
$$

Note that the dummies $g$ range over $M$ and the dummies $x$ range over $L$. Note further that functional composition in $M$ is monotonic in both arguments, as is required for the fixpoint expressions to be defined.

We list some theorems concerning the functions cl and int. Universal quantification over the free variables is left understood.

$$
\begin{aligned}
& \text { cl. } f \circ h=(\mu g:: f \circ g \sqcup h), \\
& \text { int. } f \circ h=(v g:: f \circ g \sqcap h) .
\end{aligned}
$$

In the following list we use the underscore as a sloppy notation for functional abstraction.

$$
\begin{aligned}
& f \text { is a closure function } \equiv f=\mathbf{c l} . f, \\
& \text { cl. } f \text { is a closure function (on } L), \\
& \text { cl. } f \circ \text { - is a closure function (on } M \text { ), } \\
& \text { cl is a closure function (on } M \text { ), } \\
& f \text { is an interior function } \equiv f=\text { int. } f \text {, } \\
& \text { int. } f \text { is an interior function (on } L \text { ), } \\
& \text { int. } f \circ-\text { is an interior function (on } M \text { ), } \\
& \text { int is an interior function (on } M \text { ). }
\end{aligned}
$$

We use the notions of ' $\sqcup$-junctivity' and ' $\sqcap$-junctivity' as straightforward generalizations of the notions of 'disjunctivity' and 'conjunctivity' from [DiS90, Chapter 6]. The proof of theorem (52) from Chapter 8 of that book, is easily adapted to yield a proof of:

$$
\begin{aligned}
& \text { cl. } f \text { is as } \sqcup \text {-junctive as } f \text {, } \\
& \text { int. } f \text { is as } \sqcap \text {-junctive as } f \text {. }
\end{aligned}
$$

\section{Formal Semantics of DUALITY}

One can take various points of view with respect to the rôle of predicate transformers in program semantics. We highlight some of them because it is important to appreciate the difference.

In his thesis, Lukkien takes up a purely descriptive position. He defines statements operationally and subsequently defines his predicate transformers by means of their operational interpretation. Consequently, he has to prove the properties that his predicate transformers enjoy w.r.t the program constructors.

In [DiS90], Dijkstra and Scholten work from a more prescriptive position. They hold the view that the predicate transformers actually define the statements. 
Hence, the program constructors are defined by postulating how their predicate transformers are derived from those of the parts. Subsequently, Dijkstra and Scholten argue that the standard implementation is consistent with the intended operational interpretation of the predicate transformers.

In [BvW90], Back and von Wright take an even more prescriptive point of view. For them, a program (fragment) is just a specification, albeit a specification that happens to be executable. A specification, in turn, is just a (monotonic) predicate transformer. Thus, for Back and von Wright, programs are predicate transformers and, irrespective of operational interpretability, every well-defined operator on predicate transformers can be used as 'program' constructor.

For defining the language DUALITY, we take a position somewhere between the last two above. We consider the 'processes', written in DUALITY, to be descriptions of predicate transformers. Like Dijkstra and Scholten, we maintain a distinction between the processes and their semantics (which is a predicate transformer). Like Back and von Wright, we do not restrict ourselves to constructors with an operational interpretation. We had very little choice in taking this position since our processes actually only serve as descriptions of predicate transformers and we simply needed the nonoperational constructors in our analysis of UNITY.

Below, we give a formal definition of DUALITY. The reader who wants to be able to interprete the processes operationally, should now first read appendix A, where we give an operational model for the language in terms of games. It should be stressed, however, that this operational interpretation has been constructed a posteriori and has no significance whatsoever; at best, it is fun and a source for vocabulary.

One aspect of a DUALITY process is that it is tied to some state space. This state space is set up by a collection of declarations that defines its coordinates. We assume the state space to be given and we use the everywhere-operator ' $[\ldots]$ ' from [DiS90] to quantify universally over it. We take the view that strong predicates are 'small', i.e. $p \leq q \equiv[p \Rightarrow q]$ for all predicates $p$ and $q$. Thus, for any monotonic predicate transformer $f, \boldsymbol{\mu} f$ is the strongest fixpoint and $\boldsymbol{v} f$ is the weakest one.

Another aspect of a DUALITY process is that it is built on top of a set Stat of standard statements operating on the given state space. We are not going to be specific about the elements of Stat but anything between 'move-brackets'-i.e. $[\ldots]$ or $[\ldots]-$ is assumed to be such an element. The elements of Stat will typically be simple statements, like (conditional) assignments, and we assume them to be known by their weakest-liberal-preconditions (wlp).

The semantics of a DUALITY process $s$ is given by a binary predicate tranformer $\langle s\rangle$ which should satisfy:

$$
\frac{\text { healthiness requirement: }}{\langle s\rangle \text { is monotonic in both arguments. }}
$$

We use this predicate transformer in infix notation and it is given a binding power which is lower than that of $\neg$ and higher than that of the binary logical operators.

Two processes are considered equal if they have the same semantics, i.e.

$$
s=t \equiv\langle s\rangle=\langle t\rangle .
$$

Hence, it might be considered somewhat superfluous to distinguish between a DUALITY process and its formal semantics. The reader is free to identify the 
two and see DUALITY as a language for the construction of monotonic binary predicate transformers.

For the basic ingredients of the language we define the semantics directly and for the constructors we define the whole in terms of the parts. The verification of (the preservation of) the healthiness requirement is left to the reader. Universal quantification over the predicates $p$ and $q$ is left understood.

The process nix is defined by:

$$
p\langle\text { nix }\rangle q=q \text {. }
$$

For any $S \in S t a t$ the processes $\lfloor S\rfloor$ and $\lceil S\rceil$ are given by:

$$
\begin{aligned}
& p\langle\lceil S\rceil\rangle q=p \vee \text { wlp.S.q 'demonic move'. } \\
& p\langle\lfloor S\rfloor\rangle q=p \wedge \neg \text { wlp.S. }(\neg q) \quad \text { 'angelic move' }
\end{aligned}
$$

For any two processes $s$ and $t$ the process $s ; t$ is given by:

$$
p\langle s ; t\rangle q=p\langle s\rangle(p\langle t\rangle q) \quad \text { 'composition'. }
$$

With, for all $i$ in some understood range, s.i being a process, we have:

$$
\begin{array}{ll}
p\langle\square i:: s . i\rangle q=(\forall i:: p\langle s . i\rangle q) & \text { 'demonic choice' } \\
p\langle\diamond i:: s . i\rangle q=(\exists i:: p\langle s . i\rangle q) & \text { 'angelic choice' }
\end{array}
$$

For any process $s$ we have the processes $\nabla S$ and $\Delta S$ defined by:

$$
\begin{array}{ll}
p\langle\Delta s\rangle q=(v y:: p\langle s\rangle y \wedge q) & \text { 'demonic repetition'. } \\
p\left\langle{ }_{v}\right\rangle q q=(\boldsymbol{\mu} x:: p\langle s\rangle \times \vee q) & \text { 'angelic repetition' }
\end{array}
$$

In principle, this is all there is to DUALITY. If so desired, the quantifiers $\diamond$ and [ can also be used in infix notation. The order of increasing binding power for the operators is 'choices', 'composition' and 'repetitions', where the angelic and the demonic version of an operator have the same binding power.

Remark. If we forget about the angelic constructs, we can interpret the language via the identification

$$
p\langle s\rangle q=\text { wlev.s.p.q. }
$$

The process nix now corresponds to a statement that is so empty it does't even have intermediate states. The demonic move brackets play the rôle of atomicity brackets. Sequential composition is just the one we all know. The demonic choice is the ordinary nondeterministic choice and the demonic repetition corresponds to 'repeat an arbitrary number of times'.

When using DUALITY to studie UNITY, the above identification of the semantics of a DUALITY process with Lukkien's wlev is still, more or less, appropriate. However, in the presence of the angelic constructs - and we need those - one should not rely too heavily on such interpretations.

It should be noted that, using again the underscore for functional abstraction, we can rewrite some of the language definitions without the second argument, and obtain:

$$
\begin{aligned}
& p\langle\text { nix }\rangle_{-}=i d \\
& p\langle s ; t\rangle_{-}=\left(p\langle s\rangle_{-}\right) \circ\left(p\langle t\rangle_{-}\right) \\
& p\left\langle v_{v}\right\rangle_{-}=\operatorname{cl} .\left(p\langle s\rangle_{-}\right) \\
& p\langle\Delta s\rangle_{-}=\text {int. }\left(p\langle s\rangle_{-}\right)
\end{aligned}
$$


For later reference, we list some immediate consequences of the last two equations - see (8) and (9) -

$$
p\langle\nabla s\rangle_{-} \text {is a closure function, }
$$

$p\langle\Delta s\rangle_{-}$is an interior function,

$\langle\nabla s\rangle$ is as disjunctive in its second argument as $\langle s\rangle$,

$\langle\Delta s\rangle$ is as conjunctive in its second argument as $\langle s\rangle$,

If the understood range of $i$ in (15) happens to be empty, the right hand sides become false and true respectively. Thus we obtain that

$$
\begin{aligned}
& (\diamond i: \text { false }: s . i)=\perp \text { and } \\
& (\square i: \text { false }: \text { s. } i)=\mathrm{T},
\end{aligned}
$$

with the processes $\perp$ ('lost') and $T$ ('saved') satisfying:

$$
\begin{aligned}
& p\langle\perp\rangle q=\text { false and } \\
& p\langle T\rangle q=\text { true }
\end{aligned}
$$

The reader is free to take either (18) or (19) as definition of these processes.

We conclude this section with the introduction of a notational convenience. We will frequently be interested in expressions of the shape $p\langle s\rangle$ false or false $\langle s\rangle q$. In order to keep the numerous occurrences of false from becoming 'optically dominant' we shrink it and define:

$$
p\langle s\rangle \cdot=p\langle s\rangle \text { false and } \cdot\langle s\rangle q=\text { false }\langle s\rangle q .
$$

\section{The Duality of DUALITY}

Most of the DUALITY constructs come in pairs, an 'angelic' operator and a corresponding 'demonic' one. Now, let us define, for any process $s$, the 'dual' $\sim s$ by:

$$
p\langle\sim s\rangle q=\neg(\neg p\langle s\rangle \neg q)
$$

Then the operator ' $\sim$ ' distributes over the syntax of DUALITY, replacing in the process each angelic operator by its demonic counterpart and vice versa:

$$
\begin{aligned}
& \sim \text { nix }=n i x \\
& \sim\lfloor S\rfloor=\lceil S\rceil \\
& \sim\lceil S\rceil=\lfloor S\rfloor \\
& \sim(s ; t)=\sim s ; \sim t \\
& \sim(\diamond i:: s . i)=(\square i:: \sim s . i) \\
& \sim(\square i:: s . i)=(\diamond i:: \sim s . i) \\
& \sim \nabla S=\Delta \sim S \\
& \sim \Delta S=\quad \sim \sim S \\
& \sim \sim S=s .
\end{aligned}
$$

Note that the last equality makes some of the preceding ones redundant.

We will have no use for angelic moves; they have been introduced for the sole purpose of establishing the full symmetry displayed above. We leave the verification of the equalities to the reader; for those involving the repetitions we suggest the use of the fixpoint inversion rule (3). 
Apart from the fact that the envisaged application area is UNITY, it is the symmetry that is displayed by (22) that has given DUALITY its name.

\section{A UNITY Program Written in DUALITY}

The programming formalism UNITY is developed by Chandy and Misra for the design of parallel programs [ChM90]. For reasoning about UNITY programs, Chandy and Misra supply a logic which, like Hoare-logic, is centred around relations on predicates. In [JKR89], Jutla, Knapp, and Rao introduce a collection of predicate transformers that is intended as a replacement of UNITY-logic with its relations. In this section, we construct a DUALITY process such that the predicate transformers of Jutla, Knapp, and Rao describe its semantics. We start with a very brief introduction into UNITY.

A UNITY program consists, in essence, of two parts, called the 'declare section' and 'assign section' respectively. The declare section contains the declarations of the program variables and the assign section is a set of statements operating on these variables.

From now on we assume some UNITY program to be given and we use Assign to stand for its assign section.

The declare section defines the state space and, as usual, we consider this to be no concern of us. Chandy and Misra impose some rather strong restrictions on the assign section, viz.

- Assign is nonempty,

- Assign is finite,

- Assign contains 'guarded assignments' only.

In this paper we use the first restriction once (implicitly) and we use the second restriction not at all. The only consequence of the last restriction we exploit is that the elements of Assign are 'always terminating', i.e. formally:

- wp. $S=$ wlp.S for all $S \in$ Assign

The operational interpretation of a UNITY program is that its execution consists of an infinite sequence of steps, subject to the restrictions that:

- each step consists of the execution of a statement from Assign,

- in the course of the entire computation, each statement in Assign is executed infinitely often.

The latter of these restrictions is called 'the fairness requirement' and it is doing justice to this fairness requirement that makes UNITY hard.

In [JKR89] the authors introduce some predicate transformers intended to be used as tools for reasoning about what happens in the course of a computation of a UNITY program. The two most important ones are the binary predicate transformer wsafe and the unary predicate transformer wlt, which have the following interpretations:

wsafe.q.p : holds in exactly those (initial) states for which it is guaranteed that $q$ holds at least as long as $p$ does not, i.e. 'exit from $q$ ' is possible only 'via $p$ '.

wlt.p : holds in exactly those (initial) states for which it is guaranteed that $p$ will eventually hold.

From now on, $S$ and $T$ are understood to range over Assign. 
The formal definitions given in [JKR89] for the predicate transformers wsafe and wlt amount to:

$$
\begin{array}{ll}
\text { wsafe. } q . p & =(v x::((\forall S:: \text { wlp. } S . x) \wedge q) \vee p) \\
\text { wlt. } p & =(\mu y::(\exists T:: \text { wsafe. }(\text { wlp. } T \cdot y) \cdot y) \vee p)
\end{array}
$$

Showing the correspondence between these formal definitions and the above interpretations falls outside the scope of this paper.

In [JKR89], the authors use wp instead of wlp. Since the elements of Assign are required to be finite, this yields the same result. However, the use of wp falsely suggests that the possibility of nontermination of the elements of Assign is taken into account.

The definition of wlt in [JKR89] is made more palatable with the aid of the abbreviations

$$
\begin{aligned}
& \text { stp. } T \cdot p=\text { wsafe.(wlp. } T \cdot p) \cdot p \\
& \text { we. } p=(\exists T:: \text { stp. } T \cdot p),
\end{aligned}
$$

which enable the more succinct definition

$$
\text { wlt. } p=(\boldsymbol{\mu} y:: \text { we. } y \vee p) \text {. }
$$

Note that, since (27) states that wlt $=$ cl.we, it follows immediately that wlt is a closure function.

One of the advantages of working with DUALITY is that it enables us to write down predicate-transformer-valued expressions, thus doing away with the need to baptize all the predicate transformers used.

There are several reasons for not wanting to introduce too many names. Firstly the task of finding mnemonically sensible names becomes quite taxing if we need a lot of them and if the differences in the concepts covered are subtle. Secondly, the use of lots of different concepts gives the impression that the heart of the matter has somehow been missed. Finally, the more definitions we have, the more time, ink, and mental effort we have to spend on (the trivial consequences of) unfolding them.

One of the main goals of this paper is to show that each of the predicate transformers above corresponds to the semantics of a subexpression of the DUALITY process unity given by:

$$
\text { unity }=\nabla(\diamond T:: \Delta(\square S::\lceil S\rceil) ;\lceil T\rceil) ; \perp .
$$

Thus, this process can be seen as a representation of the UNITY program in DUALITY.

Although it is, in general, difficult to interpret DUALITY processes in operational terms, we can match various ingredients of the process unity with the operational interpretation of the UNITY program:

- The part $\Delta([S::[S\rceil)$ 'implements' the repetition of steps that each consist of an execution of an arbitrary element of Assign.

- The part $\nabla(\diamond T:: \ldots ;\lceil T\rceil)$ takes care of getting each element of Assign executed 'sufficiently often', i.e. implements the fairness requirement.

- The final . . ; $\perp$ removes final states from our considerations. one.

We investigate the predicate transformers of Jutla, Knapp, and Rao one by

Ad wsafe : By rewriting the right hand side of (23) using DUALITY, we find that

$$
\text { wsafe.q. } p=p\langle\Delta \text { step }\rangle(p \vee q)
$$


where the DUALITY process step is defined by

$$
\text { step }=(\llbracket S::\lceil S\rceil) \text {. }
$$

Ad stp : Instantiating (29) with $q:=$ wlp.T.p yields

(31) $\quad$ stp. $T . p=p\langle\Delta$ step $;\lceil T\rceil\rangle p$.

Ad we : Quantifying existially over $T$ in both sides of (31), we get that

(32) we. $p=p\langle$ phase $\rangle p$

with phase defined by

$$
\text { phase }=(\diamond T:: \Delta \text { step } ;\lceil T\rceil) \text {. }
$$

Ad wlt : Using (27) and (32) we find that wlt.p $=(\boldsymbol{\mu} y:: y\langle$ phase $\rangle \vee \vee p)$ and this is unfortunate since we do not have a good handle on the duplicated dummy $y$.

There is a much more pleasant formula for wlt, but its verification requires the use of a fact that we are not ready to prove yet. Since we do want to finish the translations of this section, we insert this fact here, and the reader is kindly requested to take it on faith for the moment.

Fact

(34) $\quad-\langle$ phhase $\rangle \cdot$ is a closure function.

Using this, we can prove that

$$
\text { wlt. } p=p\langle\text { vphase }\rangle \text {. }
$$

Proof. By mutual implication

$\underline{\text { lhs } \Leftarrow \mathrm{rhs}}$ : The right hand side being a strongest fixpoint, we first observe

$$
\begin{aligned}
& {\left[p\left\langle{ }^{\prime} \text { phase }\right\rangle \cdot \Rightarrow \text { wlt. } p\right]} \\
& =\quad\{\text { definition of ' } \nabla \text { ' } \\
& {[(\boldsymbol{\mu} x:: p\langle\text { phase }\rangle x) \Rightarrow \text { wlt. } p]} \\
& \Leftarrow \quad \text { extremity } \\
& {[p\langle\text { phase }\rangle \text { wlt. } p \Rightarrow \text { wit. } p],}
\end{aligned}
$$

and we verify this, starting from the antecedent.

$$
p\langle\text { phase }\rangle \text { wlt. } p
$$

$\Rightarrow \quad\{$ wlt is a closure and -hence- weakening, monotonicity

wlt.p 〈phase $\rangle$ wlt.p

$\Rightarrow \quad\{(32)$ and pred. calc. $\}$

$$
\text { we.(wlt. } p) \vee p
$$

$=\{(27)$, folding $\}$

wlt.p.

$\underline{\text { lhs } \Rightarrow \text { rhs: }}$ We introduce the abbreviation $q$ for $p\left\langle{ }_{\nabla} p h a s e\right\rangle \cdot$ and observe

$$
\begin{array}{ll} 
& {[\text { wlt. } p \Rightarrow q]} \\
\Leftarrow & \{(27), \text { extremity }\} \\
= & {[\text { we. } q \vee p \Rightarrow q]} \\
& \{[p \Rightarrow q] \text { on account of }(34)\} \\
& {[\text { we. } q \Rightarrow q]}
\end{array}
$$




$$
\begin{array}{ccc}
\Leftarrow & \{(32), \text { reflexivity }\} \\
& q\langle\text { phase }\rangle q=q \\
\Leftarrow & \left\{(1), \text { (un-)folding with } f, p:=q\langle\text { phase }\rangle_{-}, q\right\} \\
& (\mu x:: q\langle\text { phase }\rangle x)=q \\
= & \{\text { definition of ' } \vee \text { ' }\} \\
& q\langle\text { phase }\rangle=q \\
= & \{-\langle\text { phase }\rangle \cdot \text { is idempotent on account of (34) }\} \\
& \text { true } \quad \square
\end{array}
$$

Since, from definition (27), it is immediately apparent that wlt is a closure, it follows that (34) is a necessary condition for (35) to be possible. One of the major disappointments in the course of our explorations has been that (34) is so hard to get.

The processes step and phase introduced in this section are subprocesses of the process unity defined in (28). We can now express the latter in terms of phase by

$$
\text { unity }={ } \text { phase } ; \perp .
$$

Using this and (35) we easily obtain that

$$
\text { wit. } p=p\langle\text { unity }\rangle q \text {. }
$$

Summarising the results from this section we have found that, in terms of the DUALITY processes step, phase, and unity defined by

$$
\begin{aligned}
& \text { step }=(\square S::\lceil S\rceil), \\
& \text { phase }=(\diamond T:: \Delta \text { step } ;\lceil T\rceil), \text { and } \\
& \text { unity }=\text { pphase } ; \perp,
\end{aligned}
$$

we can express the predicate transformers from Jutla, Knapp, and Rao as

$$
\begin{array}{ll}
\text { wsafe. } q . p & =p\langle\Delta \text { step }\rangle(p \vee q), \\
\text { stp. } T . p & =p\langle\Delta \text { step } ;\lceil T\rceil\rangle p, \\
\text { we. } p & =p\langle\text { phase }\rangle p, \\
\text { wlt. } p & =p\langle\text { vphase }\rangle \cdot \\
& =p\langle\text { unity }\rangle q .
\end{array}
$$

This substantiates our claim that the process unity contains everything we want to know about the UNITY program. What remains, is the obligation to establish the validity of fact (34). We meet this obligation in Section 7 but, first, we return our attention to DUALITY in general.

\section{DUALITY: The Lattice}

As already noted below (11), we can identify DUALITY processes with binary predicate transformers. As usual, we lift the order of the 'result type' to functions by universal quantification over the arguments.

$$
s \leq t \equiv(\forall p, q::[p\langle s\rangle q \Rightarrow p\langle t\rangle q]),
$$

It sometimes pays to eliminate the arguments $p$ and $q$ from our consideration and the rest of this section will be devoted to some 'argumentless' facts. 
(39) DUALITY is, with the order defined by (38), a complete and completely distributive lattice. In this lattice, the operators ' $\square$ ' and ' $\diamond$ ' denote infimum and supremum respectively. The constants $\perp$ and $T$ denote the minimum and maximum process respectively.

Lemma (39) is nice: complete lattices are pleasant to work with and, in this context, the choice operators become quite elementary.

Investigating the 'argumentless' properties of the composition, we find:

(40) (i) ';' is associative,

(ii) nix is the (left and right) identity of ';',

(iii) ';' is universally $\diamond$-junctive and [ ]-junctive in its first argument,

(iv) ';' is monotonic w.r.t. ' $\leq$ ' in both arguments.

This shows that both DUALITY and its lattice-theoretical dual (the 'upside down' version), are semi-regular algebras in the sense of [BaW92].

The repetitions can be defined in argumentless fashion:

(41) for any $s$

(i) $\quad \nabla s=(\boldsymbol{\mu} x:: s ; x \diamond n i x)$

(ii) $\Delta s=(v x:: s ; x[n i x)$

Note that the dummy $x$ in the right hand sides ranges over DUALITY processes and that the fixpoint operators denote least and largest fixpoints w.r.t. the order ' $\leq$.

Now, (41) looks familiar. Comparing (41) with (5) and (6), the following facts should come as no surprise (see also the remark below (21); see also [BaW92]).

(42) for any $s$ and $t$

(i) $\quad{ }_{\nabla} s ; t=(\mu x:: s ; x \diamond t)$ and

(ii) $\Delta s ; t=(v x:: s ; x[t)$.

(43) For any process $s$

(i) $\nabla s ;-$ and $\nabla$ are closure functions on DUALITY processes and

(ii) $\Delta s ;-$ and $\Delta$ are interior functions on DUALITY processes.

We conclude this section with a theorem about the processes step and phase from the previous section:

(44) phase $=\Delta$ step ; phase.

\section{Proof.}

$$
\begin{aligned}
& \text { phase }=\Delta \text { step ; phase } \\
= & \{\Delta \text { step } ; \text { - is contracting by }(43 . i i) \text {, hence ' } \geq \text { ' }\} \\
& \text { phase } \leq \Delta \text { step ; phase } \\
\Leftarrow \quad & \{(42 . i i), \text { extremity }\} \\
& \text { phase } \leq \text { step ; phase [ phase } \\
= & \{\text { lattice calc. }\} \\
& \text { phase } \leq \text { step } ; \text { phase }
\end{aligned}
$$

and this we prove by observing that

step ; phase 


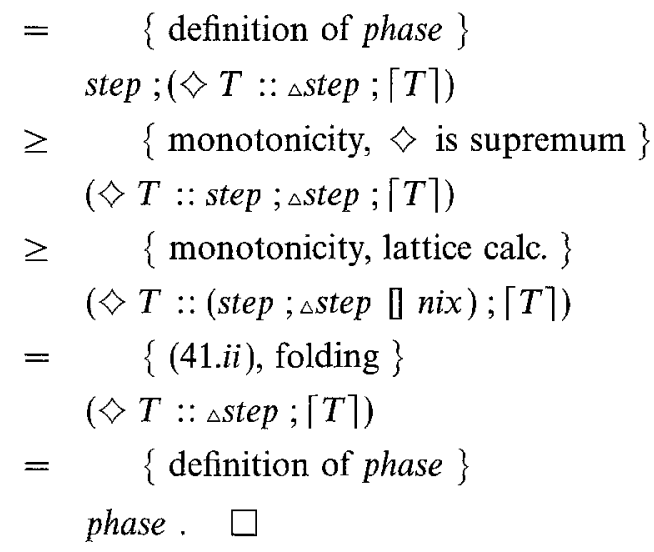

\section{Initial Safety}

One of the properties of the process vphase that we need to know for the

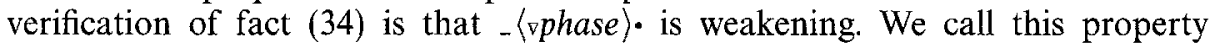
'initial safety' and in this section we study it in general. Thus, initial safety is a property that a DUALITY process may enjoy and it is given by:

(45) $\quad s$ is initially safe $\equiv(\forall p::[p \Rightarrow p\langle s\rangle \cdot])$.

On account of monotonicity we could equally well have written

(46) $s$ is initially safe $\equiv(\forall p, q::[p \Rightarrow p\langle s\rangle q])$.

which is occasionally more handy.

The initial safety of a process can easily be deduced via induction over the syntax:

(47) Initial safety inheritance

(i) Demonic moves are initially safe.

(ii) If $s$ is initially safe, so is $s ; t$.

(iii) If, for all $i$, s.i is initially safe, so is ([ $i:: s . i$ ).

(iv) If there exists an $i$ such that $s . i$ is initially safe, so is ( $\diamond i:: s . i)$.

(v) If $s$ and $t$ are initially safe, so is $\Delta s ; t$.

(vi) If $s$ is initially safe, so is $\nabla S$.

Examining the processes featuring in Section 4 we now find that:

(48) all of the proccesses $\lceil S\rceil$, step, $\Delta$ step; $\lceil T\rceil$, phase, and $\nabla$ phase are initially safe.

Note that the initial safety of phase depends upon Assign being nonempty. This is the only place where we exploit that fact. Note further that, with the initial safety of $\nabla$ phase, we are one step closer to the fulfillment of our pending obligation to prove (34). 


\section{UNITY Once More}

We refocus on the processes involved in our analysis of UNITY. Our target for this section is the verification of fact (34). Before we can do so, we need one more property of the processes $\triangle$ step and phase.

$$
\left.\frac{\text { Absorption theorem }}{[(p\langle\Delta \text { step }\rangle q)\langle\text { ophase }\rangle} q \Rightarrow p\langle\text { pphase }\rangle q\right] \text { for all } p \text { and } q \text {. }
$$

The proof of this theorem can be found in Appendix B. The reason for not including it here is that it is quite long.

We are now ready to meet our pending obligation to prove fact (34). We have to show that $-\langle\nabla$ phase $\rangle \cdot$ is monotonic, weakening, and idempotent. We already know the first two of these properties - by (10) and (48) respectively - , thus what remains to be proved is the idempotency:

$$
(p\langle{ } \text { phase }\rangle \cdot)\langle v \text { phase }\rangle \cdot=p\langle\text { pphase }\rangle \cdot \text { for all } p \text {. }
$$

Proof. We introduce the abbreviation $q$ for $p\langle\nabla$ phase $\rangle$. and now first observe that

(*) $\quad q=p\langle\Delta s t e p\rangle q$

$$
\begin{aligned}
& =\quad\{q=p\langle\text { phase }\rangle q \text { by unfolding }\} \\
& p\langle\text { phase }\rangle q=p\langle\Delta s t e p\rangle(p\langle\text { phase }\rangle q) \\
& =\quad\{\text { composition, phase }=\Delta \text { step } ; \text { phase by (44) }\} \\
& \text { true }
\end{aligned}
$$

Our proof obligation is $q\langle\nabla$ phase $\rangle \cdot=q$ and we show this by cyclic implication:

$$
\begin{aligned}
\Rightarrow & \{\text { pphase is initially safe by (48) }\} \\
& q\langle\nabla \text { phase }\rangle \\
\Rightarrow & \{\text { monotonicity }\} \\
& q\langle\nabla \text { phase }\rangle q \\
= & \{(*)\} \\
& (p\langle\Delta \text { step }\rangle q)\langle\nabla \text { phase }\rangle q \\
\Rightarrow & \{(49)\} \\
& p\langle\text { vphase }\rangle \\
= & \{p\langle\nabla \text { phase }\rangle \text { - is a closure function - see }(17)-\text { and hence idempotent }\} \\
& q . \quad \square
\end{aligned}
$$

This concludes our proof of (34) and thus closes the gap in our demonstration of the fact that DUALITY is sufficient to capture the concepts that are deemed important in UNITY. In order to demonstrate that it is also adequate, we will use DUALITY for an investigation of UNITY. The main theorems in the UNITY theory have the exploitation of 'safety properties' as their topic and our next step is to introduce such a notion in DUALITY. 


\section{Conditional Stability}

Conditional stability is a property that a predicate $r$ may enjoy with respect to a predicate $p$ in the context of a DUALITY process $s$. This state of affairs is denoted by $r$ until $p$ in $s$ and defined by:

$$
r \text { until } p \text { in } s \equiv(\forall q::[r \wedge p\langle s\rangle q \Rightarrow(r \wedge p)\langle s\rangle(r \wedge q)]) .
$$

In general it is quite hard to attach 'meaning' to a DUALITY process and consequently it is equally hard to interpret the property $r$ until $p$ in $s$. We will, however, primarily be interested in this property with subprocesses of unity for $s$, and in those cases the operational interpretation of the property is more or less what the chosen keywords suggest.

For an important class of processes, the condititional stability can be expressed much more succinctly:

(52) If $s$ is initially safe and $\langle s\rangle$ is universally conjunctive in both arguments then:

$$
r \text { until } p \text { in } s \equiv[r \Rightarrow p\langle s\rangle r] .
$$

The proof of (52) is left to the reader.

One of the more pleasant aspects of conditional stability is that it is preserved by all language constructs of DUALITY. More precisely:

$$
\text { Conditional stability inheritance: }
$$

$$
r \text { until } p \text { in } s \wedge r \text { until } p \text { in } t \Rightarrow r \text { until } p \text { in } s ; t
$$

$$
\begin{aligned}
(\forall i:: r \text { until } p \text { in } s . i) \Rightarrow & r \text { until } p \text { in }(\square i:: s . i) \\
& \wedge r \text { until } p \text { in }(\diamond i:: s . i)
\end{aligned}
$$

$$
\begin{aligned}
r \text { until } p \text { in } S \Rightarrow & r \text { until } p \text { in } \triangle S \\
& \wedge r \text { until } p \text { in } \nabla S
\end{aligned}
$$

The proofs of (i) and (ii) are straightforward. Of (iii) we prove, from the antecedent, the second conjunct of the consequent; the proof of the first conjunct is similar.

Proof. Assume $r$ until $p$ in $s$. We have to prove, for all $q$, that

$$
[r \wedge p\langle\nabla s\rangle q \Rightarrow(r \wedge p)\langle\nabla s\rangle(r \wedge q)] .
$$

Let $q$ be arbitrary and let $x$ be an abbreviation for $(r \wedge p)\langle\nabla s\rangle(r \wedge q)$, then we observe:

$$
\begin{aligned}
& {[r \wedge p\langle\nabla s\rangle q \Rightarrow x] } \\
= & \{\text { shunt }\} \\
& {[p\langle\nabla s\rangle q \Rightarrow(r \Rightarrow x)] } \\
\Leftarrow & \{\text { extremity }\} \\
= & {[p\langle s\rangle(r \Rightarrow x) \vee q \Rightarrow(r \Rightarrow x)] } \\
& \{\text { shunt }\} \\
& {[r \wedge(p\langle s\rangle(r \Rightarrow x) \vee q) \Rightarrow x] . }
\end{aligned}
$$

We prove the last line, starting from its antecedent:

$$
\begin{gathered}
r \wedge(p\langle s\rangle(r \Rightarrow x) \vee q) \\
=\quad\{\text { distribution }\}
\end{gathered}
$$




$$
\begin{array}{cc} 
& (r \wedge p\langle s\rangle(r \Rightarrow x)) \vee(r \wedge q) \\
\Rightarrow \quad & \{r \text { until } p \text { in } s\} \\
& (r \wedge p)\langle s\rangle(r \wedge(r \Rightarrow x)) \vee(r \wedge q) \\
\Rightarrow \quad & \{\text { pred. calc., monotonicity }\} \\
& (r \wedge p)\langle s\rangle x \vee(r \wedge q) \\
= & \{\text { definition of } x, \text { folding }\} \\
& x . \quad \square
\end{array}
$$

For any set $\mathscr{S}$ of DUALITY processes we define $\mathscr{L} \mathscr{S}$ (the language generated

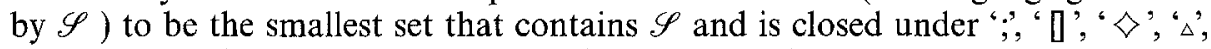
and ' $\nabla$ '. Now, theorem (53) is subsumed by its immediate consequence:

$$
\begin{aligned}
& \text { For all } r, p, \text { and } \mathscr{S} \\
& (\forall s: s \in \mathscr{P}: r \text { until } p \text { in } s) \equiv(\forall s: s \in \mathscr{L} \mathscr{S}: r \text { until } p \text { in } s) .
\end{aligned}
$$

Finally, it is worth noting that $\perp, T$, and nix are elements of $\mathscr{L} \mathscr{S}$ for all $\mathscr{S}$.

\section{Conditional Stability in UNITY}

All processes involved in our analysis of UNITY are elements of $\mathscr{L} . \mathscr{D}$, where $\mathscr{D}$ is defined by:

$$
\mathscr{D}=\{S: S \in \text { Assign : }\lceil S\rceil\} .
$$

With theorem (54) fresh in mind this invites us to investigate, for any predicates $r$ and $p$, the property $(\forall s: s \in \mathscr{D}: r$ until $p$ in $s)$. We first note that, from the definition of the semantics of demonic moves, we immediately obtain that

$$
\langle s\rangle \text { is universally conjunctive in both arguments for all } s \in \mathscr{D} \text {. }
$$

Moreover, from (55) and the definition of step it follows that

(56) $\langle$ step $\rangle$ is universally conjunctive in both arguments.

One now easily establishes that, for all predicates $r$ and $p$ :

(57) the following assertions are equivalent:

(i) $\quad(\forall s: s \in \mathscr{L} \mathscr{D}: r$ until $p$ in $s)$,

(ii) $\quad(\forall s: s \in \mathscr{D}: r$ until $p$ in $s)$,

(iii) $\quad(\forall s: s \in \mathscr{D}:[r \Rightarrow p\langle s\rangle r])$,

(iv) $\quad r$ until $p$ in step,

(v) $\quad[r \Rightarrow p\langle$ step $\rangle r]$.

The property of $r$ and $p$ that is expressed by any of the assertions in (57) is quite useful - see $(57 . i)$ - and is easily established - see (57.iii) or (57.v). From now on we suppress the final 'in step', that is, we have

$$
\begin{aligned}
r \text { until } p & \equiv r \text { until } p \text { in step } \\
& \equiv[r \Rightarrow p\langle\text { step }\rangle r]
\end{aligned}
$$

There is yet another characterization of until that illustrates the importance of the process $\Delta$ step

(59) $r$ until $p \equiv r=p\langle\Delta$ step $\rangle r$.

The verification of (59) is left to the reader. 
Since $p\langle\Delta s t e p\rangle_{-}$is an interior function, we could borrow some more topological terminology and call its fixpoints 'open'. Doing so, (59) characterizes the predicates $r$ for which $r$ until $p$ holds as the open predicates of this topology.

Note that, from (59) and the idempotency of $p\langle\Delta s t e p\rangle_{-}$, we immediately obtain that

(60) $\quad(p(\Delta s t e p\rangle r)$ until $p$.

The following properties are easily established using (58) and (56).

(61) Properties of until

(i) $\quad(\forall i:: r$ until $p . i)=r$ until $(\forall i:: p . i)$,

(ii) $\quad(\forall i:: r . i$ until $p) \Rightarrow(\forall i:: r . i)$ until $p$,

(iii) $\quad(\forall i:: r, i$ until $p) \Rightarrow(\exists i:: r . i)$ until $p$.

Theorem (61.i) states that until is universally conjunctive in its second argument. Consequently, until is monotonic in its second argument and we have that $r$ until false $=(\forall p:: r$ until $p)$. We now define the notion of (unconditional) stability of a predicate by:

$$
\begin{aligned}
r \text { is stable } & \equiv r \text { until false } \\
& \equiv(\forall p:: r \text { until } p) .
\end{aligned}
$$

The importance of the notion of stability is illustrated by the following theorem.

(63) Substitution rule:

Let predicate $r$ be stable. Then we have

$[r \Rightarrow(p\langle s\rangle q \equiv(r \wedge p)\langle s\rangle(r \wedge q))]$

for all predicates $p$ and $q$ and any process $s \in \mathscr{L} . \mathscr{D}$.

Proof.

$$
\begin{aligned}
& (\forall p, s, q: s \in \mathscr{L} \mathscr{D}:[r \Rightarrow(p\langle s\rangle q \equiv(r \wedge p)\langle s\rangle(r \wedge q))]) \\
= & \{\text { monotonicity, pred.calc. }\} \\
& (\forall p::(\forall s: s \in \mathscr{L} . \mathscr{D} ;(\forall q::[r \wedge p\langle s\rangle q \Rightarrow(r \wedge p)\langle s\rangle(r \wedge q)]))) \\
= & \{(51),(57),(58), \text { and }(62)\} \\
& r \text { is stable } \quad \square
\end{aligned}
$$

Note that, in the above proof, we have equivalence throughout and - hence the condition that $r$ be stable is not only sufficient but also necessary.

Remark. The above substitution rule is intimately connected to the controversial 'substitution axiom' of UNITY logic.

A UNITY program usually comes equiped with a condition that is assumed to hold initially. It follows that not all points of the state space - as defined by the declarations - need be reachable in the course of execution. Now let the predicate $r$ hold in exactly those states that are reachable and let the 'restricted everywhere operator' $|[\ldots]|$ ('always') be defined by

$$
|[x]| \equiv[r \Rightarrow x] .
$$

Then $r$ is stable and, using this in conjunction with the substitution rule (63), one readily verifies that

$(*) \quad\left|\left[p \equiv p^{\prime}\right]\right| \wedge\left|\left[q \equiv q^{\prime}\right]\right| \Rightarrow\left|\left[p\langle s\rangle q \equiv p^{\prime}\langle s\rangle q^{\prime}\right]\right|$

for all predicates $p, p^{\prime}, q$, and $q^{\prime}$, and any process $s \in \mathscr{L} . \mathscr{D}$. 
Now, $(*)$ looks very much like the rule of Leibniz - i.e. 'substitution of equals for equals' - but it isn't since $|[\ldots \equiv \ldots]|$ does not express equality.

In essence, $(*)$ is a reformulation of the substitution axiom of UNITY logic and it illustrates both the soundness of this axiom and the source of the controversy. It is sound in the given form, but it becomes unsound if we replace, in the consequent, $\mid[\ldots]$ by $[\ldots]$. Given a notation which is sloppy enough - e.g. leaving the everywhere operators implicit - this replacement is invisible and the arising confusion is food for controversy.

J.L.A. van de Snepscheut has given an example, exhibiting an apparent inconsistency in UNITY logic [vdS], that exploits the subtle - and invisible - difference between 'everywhere' and 'always'. Several suggestions have been made, since then, to remedy the situation.

- Jayadev Misra [Mis90] redefines the UNITY relations using inference rules instead of equalities. The effect thereof is that all that remains of v.d. Snepscheut's counter example is a proof of false $\Rightarrow$ true, and there is obviously nothing wrong with that.

- Beverly Sanders [San91] also redefines the UNITY relations, maintaining equalities but indexing them with the 'invariant assumptions' they rely on.

- Edgar Knapp [Kna90] redefines the everywhere operator [...] to stand for the always operator $|[\ldots]|$.

To these suggestions we add our own: introduce a new operator to stand for what is actually meant, viz. the 'always operator'.

\section{Some UNITY Theorems}

There is a close correspondence between until and the relation unless from UNITY logic which is defined by

$$
r \text { unless } p=(\forall S: S \in A \operatorname{ssign}:[r \wedge \neg p \Rightarrow \text { wp.S. }(r \vee p)]) \text {. }
$$

It is straightforward to show that:

$$
\begin{aligned}
& r \text { unless } p=(r \vee p) \text { until } p \text { and } \\
& r \text { until } p=r \text { unless }(r \wedge p)
\end{aligned}
$$

In [JKR89] the authors show that the UNITY relation ' $\mapsto$ ' ('leads_to') can be defined in terms of the predicate transformer wlt by:

$$
q \mapsto p \equiv[q \Rightarrow \text { wlt. } p],
$$

i.e. wlt. $p$ is the weakest predicate that 'leads to' $p$. We are now in the position to state, in various dialects, a well-known and important theorem from UNITY and give an elegant proof.

(66) PSP-rule: for all predicates $p, q, r$, and $d$ :

(i) $\quad r$ unless $d \wedge q \mapsto p \Rightarrow(r \wedge q) \mapsto(d \vee(r \wedge p))$.

(ii) $\quad r$ until $p \Rightarrow[r \wedge$ wlt. $p \Rightarrow$ wlt. $(r \wedge p)]$,

(iii) $\quad r$ until $p \Rightarrow\left[r \wedge p\left\langle{ }_{\nabla}\right.\right.$ phase $\rangle q \Rightarrow(r \wedge p)\left\langle{ }_{\nabla}\right.$ phase $\left.\rangle(r \wedge q)\right]$.

Proof. We prove the assertions of (66) in reverse order.

Ad (iii) :

$$
\Leftarrow \begin{aligned}
& {[r \wedge p\langle\nabla \text { phase }\rangle q \Rightarrow(r \wedge p)\langle\nabla \text { phase }\rangle(r \wedge q)]} \\
& \{(51)\}
\end{aligned}
$$


$r$ until $p$ in $\nabla$ hase

$\Leftarrow \quad\{$ phase $\in \mathscr{L} . \mathscr{D},(57)$, and (58) $\}$

$r$ until $p$.

Ad (ii) : Since, by (37), wlt. $p=p\langle$ pphase $\rangle \cdot$ this follows immediately from (iii) with $\overline{q:=\text { false. }}$.

Ad (i) : Translating the premisses and the consequent, using (64) and (65), we find that we have to prove

$$
[r \wedge q \Rightarrow \text { wlt. }(d \vee(r \wedge p))]
$$

from

(a) $\quad(d \vee r)$ until $d$ and

(b) $\quad[q \Rightarrow$ wlt. $p]$.

To this end we observe:

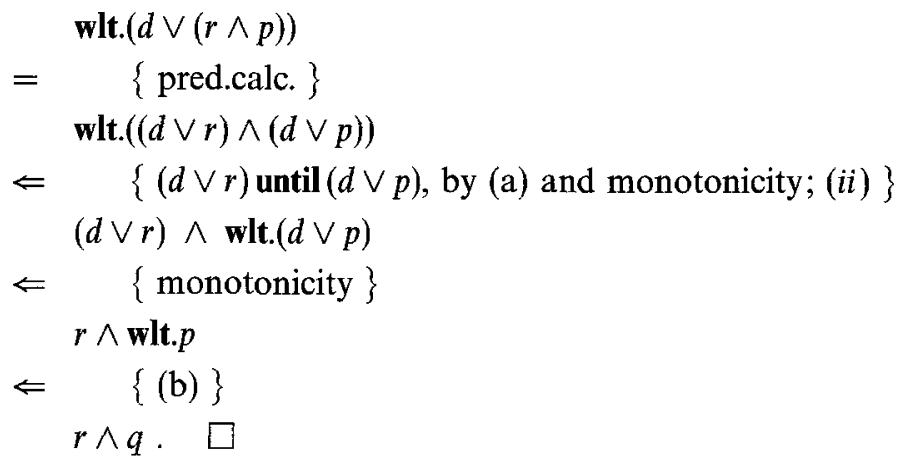

Note that (66.ii) can also be proved from (66.i) by instantiating the latter with $p, q, r, d:=p$, wlt. $p, r,(r \wedge p)$. Thus, the first two versions of the PSP-rule are equivalent, whereas the last version is somewhat more general.

In preparation of the proof of the 'completion rule' below we show that:

(67) wlt.p until $p$ for all $p$.

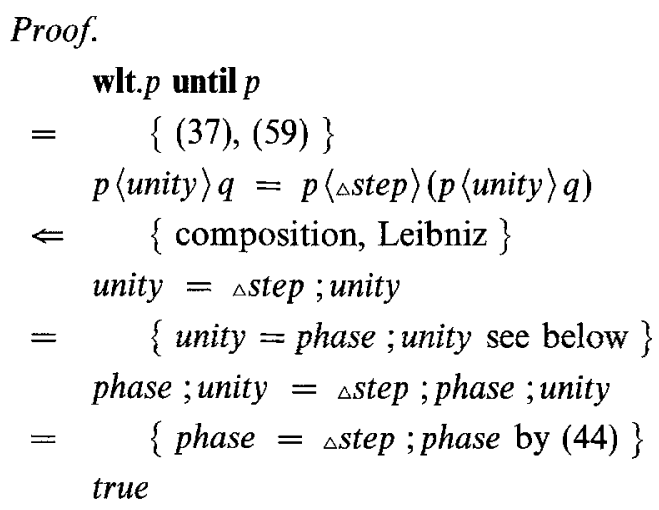

And we verify the last but one step by observing:

unity = phase ; unity

$\Leftarrow \quad\{$ (un-)folding, $\perp$ neutral element of $\diamond\}$ 


$$
\begin{aligned}
& \text { unity }=(\boldsymbol{\mu} x:: \text { phase } ; x \diamond \perp) \\
& =\quad\{(42 . i),(36)\} \\
& \text { true } \quad \square
\end{aligned}
$$

And now we are ready for the big one: the completion rule.

(68) Completion rule:

Let $B$ be a finite set of predicates such that $q$ until $p$ for all $p, q \in B$.

Then we have

$$
(\forall p: p \in B: \text { wlt. } p)=\text { wlt. }(\forall p: p \in B: p) .
$$

Proof. Since $B$ is given to be finite we can prove (68) by proving

$(\forall p: p \in C:$ wlt. $p)=$ wlt. $(\forall p: p \in C: p)$ for all $C \subseteq B$.

via induction on the cardinality of $C$. Since wlt is monotonic, ' $\Leftarrow$ ' trivially holds, and we can restrict ourselves to showing the reverse implication.

$C$ is empty:

$$
\begin{aligned}
& {[(\forall p: p \in C: \text { wlt. } p) \Rightarrow \text { wlt. }(\forall p: p \in C: p)]} \\
& =\quad\{C \text { is empty }\} \\
& \text { [true } \Rightarrow \text { wlt.true ] } \\
& =\{\text { wlt is a closure, hence weakening } \\
& \text { true. }
\end{aligned}
$$

$C$ is not empty:

From now on let $p$ and $q$ be understood to range over $C$. Thus, our demonstrandum is

$$
[(\forall p:: \text { wlt. } p) \Rightarrow \text { wlt. }(\forall p:: p)],
$$

and the induction hypothesis is

(a) $\quad(\forall p: p \neq q:$ wlt. $p)=$ wlt. $(\forall p: p \neq q: p)$ for all $q$.

Starting from the antecedent of our demonstrandum, we observe

$$
\begin{aligned}
= & (\forall p:: \text { wlt. } p) \\
& \quad(C \text { is not empty, wit is monotonic, pred.calc. }\} \\
\Rightarrow & \quad\{: \text { wlt. } p) \wedge \text { wlt. }(\exists q:: q) \\
& \text { wlt. }((\forall p:: \text { wlt. } p) \wedge(\exists q:: q)) \\
= & \{\text { pred.calc. }\} \\
& \text { wlt. }(\exists q::(\forall p:: \text { wlt. } p) \wedge q) \\
\Rightarrow \quad & \{\text { see }(\mathrm{c}) \text { below, monotonicity }\} \\
& \text { wlt. }(\exists q:: \text { wlt. }(\forall p:: p)) \\
= & \quad\{\text { range of } q \text { is nonempty, wlt is idempotent }\} \\
& \text { wlt. }(\forall p:: p) .
\end{aligned}
$$

We close the first gap by observing

(b) $\quad[(\forall p::$ wlt. $p) \wedge$ wlt. $(\exists q:: q) \Rightarrow$ wlt. $((\forall p::$ wlt. $p) \wedge(\exists q:: q))]$

$\Leftarrow \quad\{$ PSP-rule (66.ii) with $r, p:=(\forall p::$ wlt. $p),(\exists q:: q)\}$ 


$$
\begin{array}{ll} 
& (\forall p:: \text { wlt. } p) \text { until }(\exists q:: q) \\
\Leftarrow & \{(61 . i i)\} \\
& (\forall p:: \text { wlt. } p \text { until }(\exists q:: q)) \\
\Leftarrow & \{\text { until is monotonic in its second argument }\} \\
& (\forall p::(\exists q:: \text { wlt. } p \text { until } q)) \\
\Leftarrow & \{\text { instantiation with } q:=p\} \\
= & (\forall p:: \text { wlt. } p \text { until } p) \\
& \{(67)\}
\end{array}
$$

And we close the last gap by observing for any $q$

$$
\begin{aligned}
& \text { (c) } \quad[(\forall p:: \text { wlt. } p) \wedge q \Rightarrow \text { wlt. }(\forall p:: p)] \\
& \Leftarrow \quad \text { \{pred.calc. }\} \\
& {[q \wedge(\forall p: p \neq q: \text { wlt. } p) \Rightarrow \text { wlt. }(q \wedge(\forall p: p \neq q: p))]} \\
& =\{(\mathrm{a})\} \\
& {[q \wedge \text { wlt. }(\forall p: p \neq q: p) \Rightarrow \text { wlt. }(q \wedge(\forall p: p \neq q: p))]} \\
& \Leftarrow \quad \text { PSP-rule (66.ii) with } r, p:=q,(\forall p: p \neq q: p)\} \\
& q \text { until }(\forall p: p \neq q: p) \\
& =\{(61 . i)\} \\
& (\forall p: p \neq q: q \text { until } p) \\
& =\{\text { premiss of }(68)\}
\end{aligned}
$$

The name 'completion rule' is the name of a theorem in UNITY logic that looks somewhat different from the one above, viz.

Completion rule:

With dummy $i$ of some finite type we have:

$$
\begin{aligned}
\Rightarrow & (\forall i:: q . i \mapsto(p . i \vee r)) \wedge(\forall i:: p . i \text { unless } r) \\
& (\forall i:: q . i) \mapsto(\forall i:: p . i \vee r) .
\end{aligned}
$$

We used the same name for (68) because it is essentially the same theorem. One obtains (69) from (68) by instantiating with $B:=\{i:: p . i \vee r\}$, and one obtains (68) from (69) by instantiating with $i, p . i, q . i, r:=p, p$, wlt. $p,(\forall p: p \in B: p)$. We consider the relative simplicity of $(68)$ to be a strong argument for using predicate transformers rathers than relations on predicates.

\section{Chandy and Sanders' Predicate Transformers}

In [ChS] K.M. Chandy and B.A. Sanders propose another set of predicate transformers for use in reasoning about UNITY programs, viz.:

$$
\begin{aligned}
& \text { awp. } p=(\forall S: S \in \text { Assign }: \text { wlp.S.p), } \\
& \text { wst. } p=(v y:: \text { awp. } y \wedge p), \\
& \text { wto. } p=\text { wlt.(wst. } p) .
\end{aligned}
$$


We claim that these predicate transformers can be expressed in DUALITY by:

$$
\begin{aligned}
& \text { awp. } p=\cdot\langle\text { step }\rangle p \\
& \text { wst. } p=\cdot\langle\Delta \text { step }\rangle p \\
& \text { wto. } p=\cdot\langle\text { phase } ; \triangle \text { step }\rangle p .
\end{aligned}
$$

Proof. The equalities for awp and wst follow directly from the definitions and we leave their verifications to the reader. In order to verify the last equality let $q$ be an abbreviation for $\cdot\langle\Delta s t e p\rangle p$, then we observe

$$
\begin{aligned}
& \text { wto. } p=\cdot\langle\text { phase } ; \Delta \text { step }\rangle p \\
& =\quad\{\text { definitions, } q=\cdot\langle\Delta \text { step }\rangle p=\text { wst. } p\} \\
& \text { wlt. } q=\cdot\langle\text { } \text { phase }\rangle q \\
& =\{(35)\} \\
& q\left\langle{ }_{\nabla} \text { phase }\right\rangle \cdot=\cdot\langle\text { ph hase }\rangle q .
\end{aligned}
$$

We prove the last line by cyclic implication:

$$
\begin{aligned}
& q\langle\text { pphase }\rangle \\
& \Rightarrow \quad\{\text { monotonicity } \\
& q\langle\text { pphase }\rangle \\
& =\left\{\cdot\langle\Delta s t e p\rangle_{-} \text {is an interior function }- \text { see }(17)-\text { and hence idempotent }\right\} \\
& (\cdot\langle\Delta \text { step }\rangle q)\langle{ } \text { phase }\rangle q \\
& \Rightarrow \quad\{(49)\} \\
& \text { - }\langle\text { pphase }\rangle q \\
& \Rightarrow \quad\{\text { monotonicity } \\
& q\langle\nabla \text { phase }\rangle \\
& \Rightarrow \quad\{\text { vphase is initially safe } \\
& q\left\langle{ }_{p} \text { phase }\right\rangle(q(\nabla \text { phase }\rangle) \\
& =\{q\langle\nabla \text { phase }\rangle-\text { is idempotent }\} \\
& q\langle\nabla \text { phase }\rangle \cdot
\end{aligned}
$$

The process $\nabla$ phase ; $\triangle$ step has a very enjoyable property:

(72) $\quad$ phase $;$ step $=\Delta$ step $;$ vphase $; \Delta$ step .

Proof. Introducing the abbreviation $x$ for phase; $\triangle$ step our proof obligation is

$$
x=\Delta \text { step } ; x \text {. }
$$

From (42.i) with $s, t:=$ phase, $\triangle$ step we obtain, by unfolding, the only property of $x$ we'll use:

(*) $\quad x=$ phase $; x \diamond \Delta$ step.

We prove the equality by cyclic inequality:

$$
\begin{array}{cc} 
& x \\
& \quad\{\Delta \text { step } ; \text { - is contracting from }(43 . i i)\} \\
= & \{(*)\} \\
& \Delta \text { step } ;(\text { phase } ; x \diamond \Delta \text { step })
\end{array}
$$




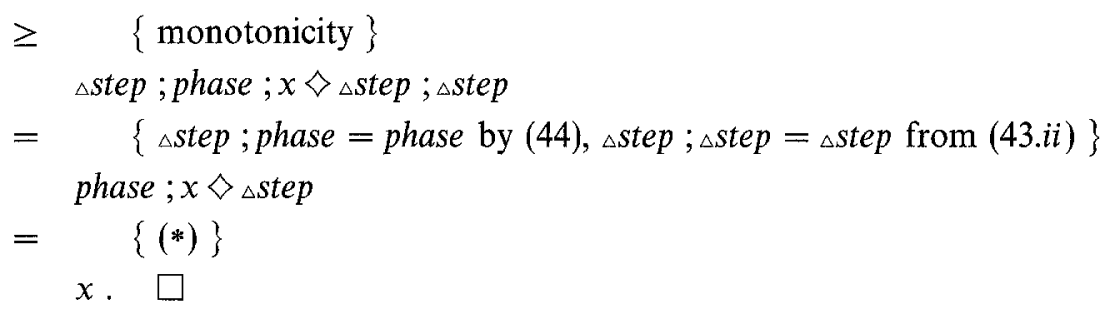

From (72) and (59) we immediately obtain that:

(73) $(p\langle$ sphase; $\Delta$ step $\rangle q)$ until $p$,

and, using that $p$ ( $\nabla$ phase _ - is idempotent, we obtain from (72) that:

(74) $\quad p\langle\text { vphase; } \Delta \text { step }\rangle_{-}$is idempotent.

Special instances of the last two theorems - with $p, q:=$ false, $p$ - give us two properties of the predicate transformer wto that Chandy and Sanders prove in their paper:

wto. $p$ is stable for all $p$ and

wto is idempotent.

One reason for Chandy and Sanders being interested in the predicate transformers they define is that, apart from being useful, these functions are conjunctive. More precisely: awp and wst are universally conjunctive and wto is finitely conjunctive. Given the equalities of (71), the conjunctive properties of awp, wst and wto are special instances of the following:

(75) $\langle$ step $\rangle$ and $\langle\Delta s t e p\rangle$ are universally conjunctive in their second arguments, 〈 ${ }$ phase; $\Delta$ step $\rangle$ is finitely conjunctive in its second argument.

Proof. From (56) and (17) we immediately obtain the universal conjunctivity $\langle$ step $\rangle$ and $\langle\Delta s t e p\rangle$ in their second arguments. Thus, what remains to be proved is that $\langle{ }$ phase ; $\Delta$ step $\rangle$ finitely conjunctive in its second argument. That is, we have to prove, for all predicates $p, q$, and $r$, that

$$
[p\langle\nabla \text { phase } ; \Delta \text { step }\rangle q \wedge p\langle\nabla \text { phase } ; \Delta \text { step }\rangle r \Rightarrow p\langle\nabla \text { phase } ; \Delta \text { step }\rangle(q \wedge r)] \text {. }
$$

To this end we introduce the abbreviations

$x$ for $p\left\langle{ }_{\nabla}\right.$ phase ; $\triangle$ step $\rangle q$ and

$y$ for $p\langle\Delta$ step $\rangle$.

Now, we first observe that, by (73) we have:

(a) $\quad x$ until $p$,

and, since $p\langle\Delta s t e p\rangle$ - is idempotent, we obtain from (59) that

(b) $y$ until $p$.

We prove our demonstrandum, starting from the antecedent:

$$
\begin{aligned}
& p\left\langle{ }_{\nabla} \text { phase } ; \triangle \text { step }\right\rangle q \wedge p\langle\nabla \text { phase } ; \Delta \text { step }\rangle r \\
& =\quad\{\text { definitions of } x \text { and } y\} \\
& x \wedge p\langle\nabla \text { phase }\rangle y \\
& \Rightarrow \quad\{(\mathrm{a}), \text { PSP-rule (66.iii) }\} \\
& (p \wedge x)\langle\nabla \text { phase }\rangle(y \wedge x) \\
& \Rightarrow \quad\{\text { monotonicity, definition of } x \text { \} }
\end{aligned}
$$




$$
\begin{aligned}
& p\langle{ } p h a s e\rangle(y \wedge p\langle\nabla p h a s e\rangle(p\langle\Delta s t e p\rangle q)) \\
& \Rightarrow \quad\{(\mathrm{b}), \text { PSP-rule (66.iii) }\} \\
& p\left\langle{ }_{\nabla} \text { phase }\right\rangle\left((p \wedge y)\left\langle{ }_{\nabla} \text { phase }\right\rangle(p\langle\Delta \text { step }\rangle q \wedge y)\right) \\
& \Rightarrow \quad\{\text { monotonicity, definition of } y\} \\
& p\left\langle{ }_{p} \text { phase }\right\rangle(p\langle{ } \text { phase }\rangle(p\langle\Delta \text { step }\rangle q \wedge p\langle\Delta \text { step }\rangle r)) \\
& =\left\{p\langle\nabla p h a s e\rangle_{-} \text {is idempotent, }\langle\Delta \text { step }\rangle \text { over } \wedge\right\} \\
& p\left\langle{ }_{\text {pphase }}\right\rangle(p\langle\Delta \text { step }\rangle(q \wedge r)) \\
& =\quad\{\text { composition }\} \\
& p\langle\nabla \text { phase } ; \Delta \text { step }\rangle(q \wedge r) \text {. }
\end{aligned}
$$

An interesting observation is that, since $p\langle\Delta s t e p\rangle \cdot=$ false, we can complicate the formula for the wlt from (37) a bit to obtain that wlt. $p=p\langle\nabla$ phase; $\Delta$ step $\rangle$. Comparing this with the formula for wto in (71), we find that both wlt and wto are special cases of the binary operator $\langle$ pphase $; \Delta s t e p\rangle$, viz. with false as right and left argument respectively.

\section{Conclusion}

The 'programming' language DUALITY is small and simple, yet rich enough to capture in one compact formula the essence of UNITY. Partly, this is of course due to the fact that DUALITY is expressly made to do so but this doesn't alter the fact that the language constructs of DUALITY are quite elementary and general. For the sequential composition this needs no elaboration and, once a complete lattice has been identified, the introduction of the two choice operators is inescapable. The repetition operators are for DUALITY what the Kleene-star is in the contexts of the relational calculus and the calculus of regular expressions. In fact, we originally wrote ' $s$ "' rather than ' $\Delta s$ ', but as the realisation dawned upon us that we also needed the dual notion, we were forced to reconsider our notation. (In the context of semi-regular algebra's, R.C. Backhouse uses the david-star ' for what we consider to be yet another Kleene-star; looking for symbols to denote our repetitions we just split this star in two.)

When one is confronted with the expression

$$
p\langle\nabla(\diamond T:: \Delta(\rceil S::\lceil S\rceil) ;\lceil T\rceil)\rangle \cdot,
$$

one might not consider this to be a 'compact' formula for wlt. $p$. However, in all fairness, the expression should be compared with the latter's definition, i.e. the horrific:

$$
(\boldsymbol{\mu} y::(\exists T::(\boldsymbol{v} x::((\forall S:: \text { wlp.S.x }) \wedge \text { wlp. } T . y) \vee y)) \vee p)
$$

or with the full unfolding of $(*)$ :

$$
(\mu y::(\exists T::(v x::(\forall S:: p \vee \text { wlp.S. } x) \wedge(p \vee \text { wlp. } T . y)))) \text {. }
$$

Next to these two monsters, the DUALITY expression looks rather harmless, yet it contains exactly the same information.

Even though we repeatedly stressed that DUALITY processes defy operational interpretation the formula

$(* *) \quad \nabla(\diamond T:: \Delta(\rceil S::\lceil S\rceil) ;\lceil T\rceil) ; \perp$

does provide a handle on operationally understanding the 'mechanism' underlying 
UNITY (see the discussion below (28)). Actually, we started our investigation with a formula that we guessed. The guessed formula was written down with a completely operational idea of what it should mean and, although it wasn't exactly the one above, it was very close. The formula that we originally wrote down was - with a prefix notation for the Kleene-star - :

$$
\infty(\diamond T:: *(\square S::\lceil S\rceil) ;\lceil T\rceil),
$$

and we read this as:

"Infinitely often, for an each time suitably chosen $T$ do:

first any finite number of times an arbitrary $S$, and then $T$."

The problem with the operator ' $\infty$ ' ( which we could now define by: $\propto s=\nabla S ; \perp$ ) was that it did not offer a good enough separation of concerns. The point is that we are not, in fact, interested in infinite computations, but in 'long enough' finite prefixes thereof. In (**), the initial ' $\mathrm{v}$ ' takes care of 'generating some long enough computation' and the final ' $; \perp$ ' eliminates the final state from our considerations. Hence, we can read (**) as:

"Often enough, for an each time ... etc."

Formula $(* *)$ is also very illuminating when we consider parallel composition. Given two UNITY programs - operating on the same state space - with assignsections $A$ and $B$ respectively, the parallel composition of these programs is the UNITY program with the assign-section $A \cup B$. One of the more disappointing aspects of UNITY is that the properties of a parallel composition are not easily deduced from those of the parts.

Now consider $(* *)$; it doesn't really help solving the problem, but goes a long way in explaining where it comes from. In $(* *)$, the assign section is the understood range of two quantifications. One of these quantifications is a demonic choice (an infimum), the other an angelic choice (a supremum), and - with some additional decoration - the former appears nested in the latter. Moderate experience in lattice theory suffices to see that, as a function of this common range, the formula can't really be expected to have many pleasant properties.

That the predicate transformers of Jutla, Knapp, and Rao be expressible in DUALITY has been one of the leading concerns in its design. It is therefore not very surprising that these predicate transformers are, indeed, expressible. Another leading concern has been generality, where we relied on our experience in relational calculus and lattice theory to identify the notions that are general.

When we turned our attention to the predicate transformers of Chandy and Sanders, DUALITY was already a finished product. We regard the fact that these predicate transformers could be expressed without much ado, as an indication that the formalism is sufficiently general.

\section{Appendices}

\section{A. An Operational Model for DUALITY}

In this section we give an operational model for the 'programming' language DUALITY. We will redefine the language constructs by stating, in operational terms, what they are supposed to mean; and we will redefine the binary predicate transformer from Section 2 by giving its interpretation in the operational model. 
Subsequently, we will argue that, for this interpretation, the postulates from Section 2 do indeed hold.

Processes in DUALITY describe nondeterministic, imperative, sequential programs. As usual, these programs operate on some state space. We assume this state space to be given and, other than that it is nonempty, we are not interested in any properties it might have.

For execution of a DUALITY process we envisage a machine that is equipped with two identical processors called 'the angel' and 'the demon' respectively. Execution of a process consists of a sequence of actions. Each of these actions has an 'owner' being the processor that has to execute it. If such an action is nondeterministic we assume that its owner is capable of making full use of the freedom offered, i.e. whatever is 'legal' is also possible. Obviously, by the way they execute their own actions, both processors influence the entire execution.

We are going to distinguish 'good' executions and 'bad' executions. Moreover, we assume that the angel always strives for a 'good' execution whereas the demon always strives for a 'bad' one. Thus, an execution can be seen as a game played by the processors, where each strives to win at the cost of the other.

\section{A.1. The Rules of the Game}

Henceforth the processors are called 'players', an execution is called a 'match' and a body of rules determining a set of (legal) matches is called a 'game'. In this section we discuss the rules. Note that the rules of a game determine the possible matches but not who wins them.

The legal matches are determined by the game that is being played. This game, in turn, is determined by two parameters, viz. the process that has to be executed and a dichotomy of the state space. This dichotomy is given by a predicate $p$ and it divides the statespace into an 'angel's half' and a (complementary) 'demon's half', where (by convention)

$p \quad$ : holds exactly on the angel's half (and thus)

$\neg p$ : holds exactly on the demon's half.

A match played by the rules as determined by a predicate $p$ and a process $s$, we call 'a match by $p \& s$ ', or, if $p$ is understood from the context, 'a match by $s$ '.

Now we come to the processes themselves. Processes are built from basic processes, using various contructors enabling us to construct new processes from existing ones. Below, we describe the rules of the basic processes and, for each of the constructors, we describe the rules of the whole in terms of those of the constituents.

The rules are such that in case of an infinite match exactly one of the players can be held responsible for the nontermination, and we call this a 'failure' of the player in question. If, in the course of a 'composite' match, one of the players fails on a submatch, it fails on the entire match.

In the course of a match, the division of the state space in halves is not subject to modification and we assume this division to be given.

nix :

This is 'the empty game'. A match by the rules of nix ends as soon as it starts, the initial state is also final. 
Moves :

A move is the only kind of action that can modify the state and it consists of one of the players executing an 'ordinary' statement. The statements should be ordinary in the sense that they specify computations for a single processor. For any ordinary statement $S$ we have the move $\lfloor S\rfloor$, owned by the angel, and the move $\lceil S\rceil$, owned by the demon, i.e. we have:

$\lfloor S\rfloor$ : the angel executes $S$,

$\lceil S\rceil$ : the demon executes $S$.

Execution of moves is subject to the restriction that:

A player is allowed to make a move only if the initial state is in its own half.

And for failures we have:

If a move takes forever - possibly because the owner is not allowed to make it - the owner of the move fails.

Moves are the only kind of action for which the halves play a rôle. Note that, the stronger $p$, the smaller the angel's half and the less freedom the angel consequently has for executing its moves. If $p=$ false the angel fails on any of its moves and the demon can only fail by producing an infinite computation.

Sequential composition :

For any two processes $s$ and $t$, the sequential composition $s ; t$ is a process. A match by $s ; t$ consists of a match by $s$ that, if finite, is followed by a match by $t$.

\section{Choices :}

Let, for all $i$ in some understood range s.i be a process. We can construct a new process, in which one of the players selects an $i$ for which the rules of s.i subsequently apply. We so obtain the processes $(\diamond i::$ s.i) and (] $i::$ s.i) where:

$(\diamond i:: s . i) \quad:$ process s.i for some $i$ chosen by the angel,

(口 $i::$ s.i) $\quad$ : process s.i for some $i$ chosen by the demon.

If a player has to choose from zero options, it fails.

Repetitions :

Let $s$ be a process. We can construct a new process such that a match by the rules of the new process consists of a (possibly empty, possibly infinite) sequence of submatches by $s$, and where one of the players is 'in control'. The player that is in control must decide, initially and after each submatch by $s$, whether or not to end the entire match. We so obtain:

$\nabla S \quad$ : a repetition of $s$ under control of the angel,

$\Delta S \quad$ : a repetition of $s$ under control of the demon.

A match that consists of an infinite sequence of (necessarily finite) submatches by $s$ is considered a failure of the player that is in control of the repetition.

Note that the player in control of a repetition of process $s$ initially faces a choice between two options: either it can decide to end the match there and then, or it can decide that $s$ should be played once and have the same choice afterwards. Expressing this somewhat more succinctly, we get 


$$
\begin{aligned}
& \nabla s=s ; \nabla s \diamond n i x \text { and } \\
& \triangle S=s ; \Delta S[n i x .
\end{aligned}
$$

This ends our description of how the games are constructed.

\section{A.2. Changing Sides}

The 'player-specific' constructs of DUALITY (i.e. moves, choices, and repetitions) come in pairs, viz. one construct for the angel and one for the demon. For any game, we can construct a new game, which is the old one with the rôles of the players interchanged.

Now, let the old game be given by $p \& s$. Since, in the new game, the players must be able to make each others moves, they should exchange halves, and the division of the state space for the new game is given by $\neg p$. Since the players should perform each others actions, the process of the new game is obtained by replacing in $s$ each of these player-specific constructs by its counterpart.

Let us now (re)define the operator ' $\sim$ ' such that $\sim s$ denotes the process $s$ with $(\lfloor\rfloor, \diamond, \nabla)$ and $([\rceil, \square, \triangle)$ interchanged. Then, the new game is given by $\neg p \& \sim s$.

Note that (22) can now be seen as defining the operator ' $\sim$ ' inductively over the syntax.

\section{A.3. Winning the Game}

The rules of a game determine the possible matches but not who wins them. In order to be able determine the winner of a match, we give a classification of matches.

Using a predicate $q$ on the state space for the classification of finite matches, we have the following exhaustive and unambiguous classification:

'finally $q$ ' : the class of finite matches for which the final state satisfies $q$, 'finally $\neg q$ ' : the class of finite matches for which the final state does not satisfy $q$,

'demon fails' : the class of infinite matches where the demon fails, 'angel fails' : the class of infinite matches where the angel fails.

Matches belonging to the class 'finally $q$ or demon fails' are won by the angel and matches belonging to the class 'finally $\neg q$ or angel fails' are won by the demon. Note that, although a player may need supernatural patience to claim its victory, there is no such thing as a draw.

We are interested in the initial states for which the angel has a winning strategy. To this end we introduce, for any process $s$, the binary predicate transformer $\langle s\rangle$, which we use in infix notation and which has the interpretation that

$p\langle s\rangle q:$ holds in exactly those initial states for which the angel can ensure that a match by $p \& s$ belongs to the class 'demon fails or finally $q$ '.

We are also interested in the initial states for which the demon has a winning strategy. Using that a match by $\neg p \& \sim s$ is a match by $p \& s$ with the rôles of the demon and the angel interchanged we get :

$\neg p\langle\sim s\rangle q \quad$ : holds in exactly those initial states for which the demon can ensure that a match by $p \& s$ belongs to the class 'angel fails or finally $q$ ', and thus we find for the conjugate $p\langle\sim s\rangle^{*} q$ (i.e. $\neg(\neg p\langle\sim s\rangle \neg q)$ ), 
$p\langle\sim s\rangle^{*} q \quad$ : holds in exactly those initial states for which the demon can not ensure that a match by $p \& s$ belongs to the class 'angel fails or finally $\neg q$ '.

Since at most one of the players can have a winning strategy we have:

$$
\left[p\langle s\rangle q \Rightarrow p\langle\sim s\rangle^{*} q\right]
$$

We will argue that the postulates $(10-16)$ of Section 2 do indeed hold for the model that we just described. For the composite processes we do so by induction on the syntax.

In parallel, i.e. also by induction on the syntax, we will argue the validity of the 'strong duality result' (21), i.e. equivalence in (77). Note that, in this section, (22) may count as the inductive definition of ' $\sim$ ' and that - see the interpretation above - (21) expresses that, in every initial state, exactly one of the players has a winning strategy.

In order to establish the validity of the healthiness condition (10) it is sufficient to observe that, the weaker $p$ and $q$, the larger the classes 'demon fails' and 'finally $q$ ' respectively, and the easier, therefore, the angel's task.

Proof. For the process nix.

(12) : nix is the empty game. Since the demon cannot fail and the initial state is the final state, we have that 'demon fails or finally $q$ ' iff 'initially $q$ '.

dualism :

$$
\begin{array}{cc} 
& p\langle\text { nix }\rangle q=p\langle\sim \text { nix }\rangle^{*} q \\
= & \{(22), \text { conjugation }\} \\
& p\langle\text { nix }\rangle q=\neg(\neg p\langle\text { nix }\rangle \neg q) \\
= & \{(12)\} \\
& q=\neg \neg q \\
= & \{\text { pred. calc. }\} \\
& \text { true } . \quad \square
\end{array}
$$

Proof. For the moves.

(13) : We observe for a demonic move:

$$
\begin{aligned}
& p\langle\lceil S\rceil\rangle q \\
& =\quad \text { interpretation }
\end{aligned}
$$


And for an angelic move

$$
=\begin{aligned}
& p\langle\lfloor S\rfloor\rangle q \\
& \{\text { interpretation }\}
\end{aligned}
$$

the angel can ensure that a match by $p \&\lfloor S\rfloor$ belongs to the class "demon fails or finally $q$,

$=\{\lfloor S\rfloor$ is a move for the angel. Thus, the demon cannot fail and if $p$ doesn't hold the angel fails \}

$p \wedge$ the angel can ensure that a match by $p \&\lfloor S\rfloor$ belongs to the class 'finally $q$ '

$=\{$ if $p$ holds, the angel executes $S\}$

$p \wedge$ there exists a computation by $S$ terminating in a state satisfying $q$

$=\{$ de Morgan $\}$

$p \wedge \neg$ (all finite computations by $S$ terminate in a state satisfying $\neg q$ )

$=\quad\{$ interpretation of wlp.S $\}$

$p \wedge \neg \mathbf{w l p . S .}(\neg q)$.

dualism : We have to prove

$$
\begin{aligned}
& p\langle\lfloor S\rfloor\rangle q=p\langle\sim\lfloor S\rfloor\rangle^{*} q \text { and } \\
& p\langle\lceil S\rceil\rangle q=p\langle\sim\lceil S\rceil\rangle^{*} q .
\end{aligned}
$$

Since, by (22), $\sim\lfloor S\rfloor=\lceil S\rceil$ and $\sim$ and * are involutions, these two are easily seen to follow from each other. We show the first only :

$$
\begin{aligned}
& p\langle\sim\lfloor S\rfloor\rangle^{*} q \\
& =\quad \text { conjugation, }(22)\} \\
& \neg(\neg p\langle\lceil S\rceil\rangle \neg q) \\
& =\{(13)\} \\
& \neg(\neg p \vee \text { wlp.S.S. }(\neg q)) \\
& =\{\text { de Morgan }\} \\
& p \wedge \neg \text { wlp.S. }(\neg q) \\
& =\{(13)\} \\
& p\langle\lfloor S\rfloor\rangle q \text {. }
\end{aligned}
$$

Proof. For sequential composition.

(14) : A match by $s$; $t$ consists of match by $s$ followed, if finite, by a match by $t$. Obviously the angel has a winning strategy iff for the first part (by $p \& s$ ) it can ensure that, if the demon doesn't fail, it ends in a state where the angel has a winning strategy for the second part (by $p \& t$ ). 
dualism :

$$
\begin{array}{cc} 
& p\langle\sim(s ; t)\rangle^{*} q \\
= & \{(22), \text { conjugation }\} \\
& \neg(\neg p\langle\sim s ; \sim t)\rangle \neg q) \\
= & \{(14)\} \\
& \neg(\neg p\langle\sim s\rangle(\neg p\langle\sim t\rangle \neg q)) \\
= & \{\text { conjugation (twice) }\} \\
= & p\langle\sim s\rangle^{*}\left(p\langle\sim t\rangle^{*} q\right) \\
= & \{\text { dualism for } s \text { and } t\} \\
= & p\langle s\rangle(p\langle t\rangle q) \\
& \{\langle(14)\} \\
& p\langle s ; t\rangle q .
\end{array}
$$

Proof. For the choices.

(15) : Trivial.

dualism : de Morgan.

Proof. For the repetitions.

(16) and dualism : As usual, repetitions give rise to very involved operational reasoning. It is here that the operational justifications of the formulae for \langle\rangle and the duality result (21) become intimately entwined. We start by observing that from the equalities in (76), using (12), (14), and (15), we obtain that:

$$
\begin{aligned}
& {[p\langle\nabla s\rangle q \Leftarrow(\boldsymbol{\mu} x:: p\langle s\rangle x \vee q)] \text { and }} \\
& {[p\langle\Delta s\rangle q \Rightarrow(\boldsymbol{v} y:: p\langle s\rangle y \wedge q)] .}
\end{aligned}
$$

Thus, what remains to be shown, is that the reverse implications hold. We begin doing so for $\Delta s$.

Introducing the abbreviation $y$ for $(v y:: p\langle s\rangle y \wedge q)$ we have to show that [ $y \Rightarrow p\langle\Delta s\rangle q$ ], i.e. given 'initially $y$ ', we have to show the existence of a winning strategy for the angel for a match by $\Delta s$. The only properties of $y$ we use are

(i) $\quad[y \Rightarrow p\langle s\rangle y]$

(ii) $[y \Rightarrow q]$

Let a match be called 'critical' if it is not of the type 'demon fails'. A match by $\Delta s$ consists of a sequence of submatches by $s$, broken off at the demon's convenience. The demon fails if the sequence is infinite or if it fails on a submatch, thus, a critical match by $\Delta s$ consists of a finite sequence of critical submatches by $s$.

In order to show that the angel has a winning strategy if $y$ holds initially, we first observe that:

$$
\begin{aligned}
\Rightarrow & y(i)\} \\
& p\langle s\rangle y \\
= & \{\text { interpretation }\} \\
& \text { the angel can ensure that a match by } s \text { belongs to the class 'demon fails or } \\
& \text { finally } y \text { ' } \\
= & \{\text { notion of 'critical' }\}
\end{aligned}
$$


the angel can ensure that a critical match by $s$ belongs to the class 'finally $y$ '. Thus, the angel has a strategy such that

for any critical match by $s$ : 'initially $y$ ' $\Rightarrow$ 'finally $y$ ', and we show that this strategy is winning for a match by $\Delta s$ :

for any critical match by $s$ : 'initially $y$ ' $\Rightarrow$ 'finally $y$ '

$\Rightarrow \quad\{y$ is kept invariant $\}$

for any finite sequence of critical matches by $s$ : 'initially $y$ ' $\Rightarrow$ 'finally $y$ '

$\Rightarrow \quad\{$ preceding observation about the critical matches by $\Delta S$ \}

for any critical match by $\Delta s$ : 'initially $y$ ' $\Rightarrow$ 'finally $y$ '

$\Rightarrow \quad\{$ notion of 'critical' $\}$

for any match by $\Delta s$ : 'initially $y$ ' $\Rightarrow$ 'demon fails or finally $y$ '

$\Rightarrow \quad\{$ by (ii) the class 'finally $y$ ' is contained in the class 'finally $q$ '

for any match by $\Delta s$ : 'initially $y$ ' $\Rightarrow$ 'demon fails or finally $q$ '

Thus, for an initial state satisfying $y$, the angel has indeed a winning strategy and it consists of 'maintaining $y$ '.

Having taken care of the equality for the demonic repetition, our remaining obligations are

(iii) $\quad p\langle\nabla s\rangle q=(\boldsymbol{\mu} x:: p\langle s\rangle \times \vee q)$

(iv) $\quad p\langle\nabla s\rangle q=p\left\langle\sim_{\nabla} s\right\rangle^{*} q$

(v) $\quad p\langle\Delta s\rangle q=p\langle\sim \Delta s\rangle^{*} q$

Of these, $(v)$ is easily proved from (iv), using (22) and the fact that $\sim$ and * are involutions. We show the first two in one go by cyclic implication:

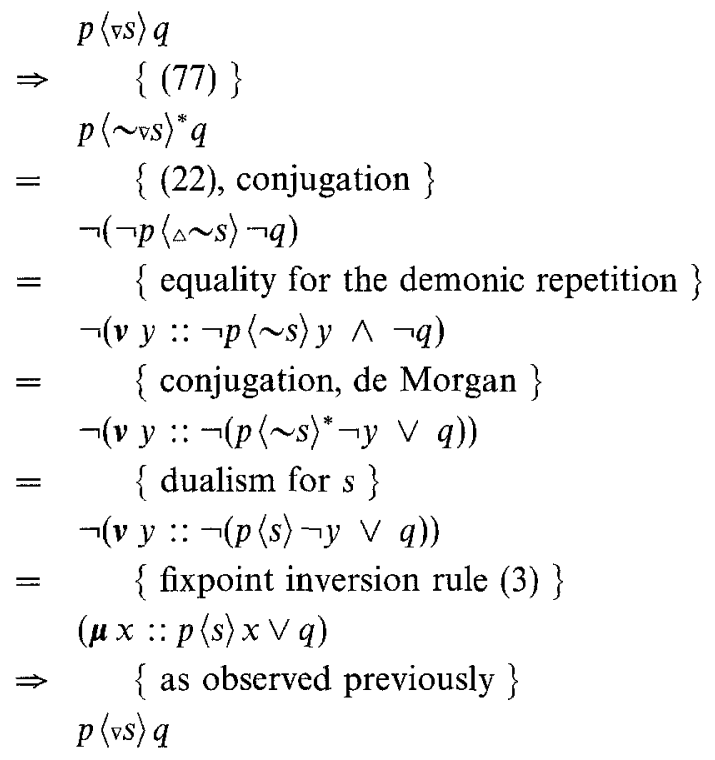

and, since the first and last line are equal, we have equality throughout. Particularly, we have equality between the first two lines - which establishes (iv) - and equality between the last two lines - which establishes (iii) -. 


\section{B. Proof of the Absorption Theorem}

The proof of the absorption theorem (49) is rather long. The main reason for this is that we need to exploit some properties of the basic constituents of the processes involved. In order to be able to do so we have to unfold the definitions all the way down to these basic constituents.

The special properties that we need are the following:

(a) $\quad p\langle\lceil S\rceil\rangle q=p \vee \cdot\langle\lceil S\rceil\rangle q$ and

(b) $\quad p\langle$ step $\rangle q=p \vee \cdot\langle$ step $\rangle q$

for all predicates $p$ and $q$ and all $S$,

and these are easily verified by inspecting the definitions.

Now, let $p$ and $q$ be arbitrary. Introducing the abbreviations

$x$ for $p\langle\Delta s t e p\rangle q$ and

$y$ for $p\langle$ ph phase $\rangle q$

our proof obligation becomes

$\left[x\left\langle{ }_{p}\right.\right.$ hhase $\left.\rangle q \Rightarrow y\right]$.

The only thing we'll use about $x$ and $y$ is that they are defined as fixpoints, i.e.

(c) $\quad x=p\langle$ step $\rangle x \wedge q$ and

(d) $\quad y=p\langle$ phase $\rangle y \vee q$.

Now, for a start, we observe

$$
\begin{aligned}
& {\left[x\left\langle{ }_{\nabla} \text { phase }\right\rangle q \Rightarrow y\right]} \\
& \Leftarrow \quad \text { \{definition of ' } \mathrm{v} \text { ', extremity }\} \\
& {[x\langle\text { phase }\rangle y \vee q \Rightarrow y]} \\
& =\{(\mathrm{d})\} \\
& {[x\langle\text { hase }\rangle y \vee q \Rightarrow p\langle p h a s e\rangle y \vee q]} \\
& \Leftarrow \quad \text { \{pred. calc. , definition of phase }\} \\
& {[x\langle\diamond T:: \Delta \text { step } ;\lceil T\rceil\rangle y \Rightarrow p\langle\diamond T:: \Delta s t e p ;\lceil T\rceil\rangle y]} \\
& \Leftarrow \quad \text { definition of ' } \diamond \text { ', pred. calc. }\} \\
& (\forall T::[x\langle\Delta \text { step } ;\lceil T\rceil\rangle y \Rightarrow p\langle\Delta \text { step } ;\lceil T\rceil\rangle y]) .
\end{aligned}
$$

We continue by proving, for any $T$, the term of this last line. Thus, with $T$ an arbitrary element of Assign and the abbreviation

$$
z \text { for } x\langle\Delta s t e p ;\lceil T\rceil\rangle y,
$$

our remaining proof obligation is

$$
[z \Rightarrow p\langle\Delta \text { step } ;\lceil T\rceil\rangle y] \text {. }
$$

Again, we use only that $z$ is a fixpoint, but we work that out somewhat further:

$$
\begin{array}{cc} 
& \text { true } \\
= & \{\text { composition }\} \\
& z=x\langle\Delta \text { step }\rangle(x\langle\lceil T\rceil\rangle y) \\
\Rightarrow \quad & \{\text { unfolding }\} \\
& z=x\langle\text { step }\rangle z \wedge x\langle\lceil T\rceil\rangle y \\
=\quad\{(\mathrm{b}),(\mathrm{a}), \text { and distribution }\}
\end{array}
$$


(e) $z=x \vee(\cdot\langle$ step $\rangle z \wedge \cdot\langle\lceil T\rceil\rangle y)$.

And now, we're ready for the final stretch:

$$
\begin{aligned}
& {[z \Rightarrow p\langle\Delta \text { step } ;\lceil T\rceil\rangle y]} \\
& \Leftarrow \quad \text { composition, extremity } \\
& {[z \Rightarrow p\langle\text { step }\rangle z \wedge p\langle\lceil T\rceil\rangle y]} \\
& =\{(\mathrm{e}), \text { pred.calc. }\} \\
& {[x \Rightarrow p\langle\text { step }\rangle z \wedge p\langle\lceil T\rceil\rangle y] \wedge[\cdot\langle\text { step }\rangle z \wedge \cdot\langle\lceil T\rceil\rangle y \Rightarrow p\langle\text { step }\rangle z \wedge p\langle\lceil T\rceil\rangle y]} \\
& =\{\text { second conjunct holds on account of monotonicity }\} \\
& {[x \Rightarrow p\langle\text { step }\rangle z \wedge p\langle\lceil T\rceil\rangle y]} \\
& \Leftarrow \quad\{\text { step } \leq\lceil T\rceil\} \\
& {[x \Rightarrow p\langle\text { step }\rangle \wedge p\langle\text { step }\rangle]} \\
& \Leftarrow \quad\{[x \Rightarrow p\langle\text { step }\rangle x] \text { from (c) }\} \\
& {[p\langle\text { step }\rangle x \Rightarrow p\langle\text { step }\rangle z \wedge p\langle\text { step }\rangle y]} \\
& \Leftarrow \quad \text { monotonicity, transitivity } \\
& {[x \Rightarrow z] \wedge[x \Rightarrow q] \wedge[q \Rightarrow y]} \\
& =\quad\{\text { from (e), (c), and (d) }
\end{aligned}
$$

And this concludes the proof.

\section{References}

[BaW92] Backhouse, R. and van der Woude, $\mathrm{J}$.: A relational theory of datatypes. In: Lecture Notes of the STOP 1992 Summerschool on Constructive Algorithmics.

[BvW90] Back, R.J.R. and von Wright, J.: Duality in specification languages: a lattice-theoretical approach. Acta Informatica, 27:583-625, 1990.

[ChM90] Chandy, K.M. and Misra, J.: Parallel Program Design, a foundation. Addison-Wesley, 1990.

[ChS] Chandy, K.M. and Sanders, B.A.: Conjunctive predicate transformers for reasoning about concurrent computation. to appear in: the Science of Computer Programming.

[DiS90] Dijkstra, E.W. and Scholten, C.S.: Predicate Calculus and Program Sernantics. Texts and Monographs in Computer Science. Springer Verlag New York Inc., 1990.

[Hes92] Hesselink, W.H.: Programs, Recursion and Unbounded Choice. Cambridge Tracts in Theoretical Computer Science. Cambridge University Press, 1992.

[Hes94] Hesselink, W.H.: Nondeterminism and recursion via stacks and games. Theoretical Computer Science, 124:273-295, 1994.

[HeR92] Hesselink, W.H. and Reinds, R.: Temporal preconditions of recursive procedures. In W.P. de Roever J.W. de Bakker and G. Rozenberger, editors, Semantics: Foundations and Applications, pages 236-260. Springer-Verlag, 1992.

[JKR89] Jutla, C. S., Knapp, E. and Rao, J. R.: A predicate transformer approach to semantics of parallel programs. In Proceedings of the 8th Annual ACM symposium on Principles of Distributed Computing, pages 249-263. ACM Press, 1989.

[Kna90] Knapp, E.: Refinement as a basis of Concurrent Program Design. PhD thesis, The University of Texas at Austin, 1990.

[Luk90] Lukkien, J.J.: Parallel Program Design and Generalized Weakest Preconditions. PhD thesis, Rijksuniversiteit Groningen, 1990.

[Mis90] Misra, J. Soundness of the substitution axiom. Notes on UNITY: 14-90, March 1990.

[San91] Sanders, B.A.: Eliminating the substitution axiom from unity logic. Formal Aspects of Computing, 3:189-205, 1991.

[vdS] van de Snepscheut, J.L.A.: Is UNITY consistent? internet newsgroup comp.theory. 